\title{
Constitutive Modeling of Anisotropic Sand Behavior in Monotonic and Cyclic Loading
}

\author{
Zhiwei Gao ${ }^{1}$ and Jidong Zhao ${ }^{2}$
}

\begin{abstract}
An anisotropic plasticity model is proposed to describe the fabric effect on sand behavior under both monotonic and cyclic loading conditions within the framework of anisotropic critical state theory. The model employs a cone-shaped bounding surface in the deviatoric stress space and a yield cap perpendicular to the mean stress axis to describe sand behavior in constant mean stress shear and constant stress ratio compression, respectively. The model considers a fabric tensor characterizing the internal structure of sand associated with the void space system and its evolution with plastic deformation. The fabric evolution law is assumed to render the fabric tensor to become codirectional with the loading direction tensor and to reach a constant magnitude of unit at the critical state. In constant stress ratio compression, the final degree of anisotropy is proportional to a normalized stress ratio. An anisotropic variable defined by a joint invariant of the fabric tensor and loading direction tensor is employed to describe the fabric effect on sand behavior in constant mean stress monotonic and cyclic shear. A systematic calibrating procedure of the model parameters is presented. Satisfactory comparison is found between the model simulations and test results on Toyoura sand in both monotonic and cyclic loadings with a single set of parameters. The important role of fabric and fabric evolution in capturing the sand behavior is highlighted. Limitations and potential improvement of the model in describing cyclic mobility of very dense sand and sand behavior in nonproportional loading have been discussed. DOI: 10.1061/(ASCE)EM.1943-7889.0000907. @ 2015 American Society of Civil Engineers.
\end{abstract}

Author keywords: Constitutive modeling; Fabric tensor; Anisotropy; Bounding surface; Cyclic loading; Sand.

\section{Introduction}

Natural and manmade sand deposits/samples are frequently crossanisotropic due to gravitational forces and/or compaction. The anisotropic soil fabric consists of preferentially orientated sand particles, interparticle contacts, and void spaces with special geometric properties, and plays an important role in affecting the overall sand behavior. For example, repeated experimental data indicate that sand fabric may significantly affect both the strength and the deformation behavior of sand. Careful consideration of fabric effects has to be a major component in safe design of major infrastructures since they are commonly built on/in sand with fabric anisotropy (Uthayakumar and Vaid 1998).

With two model strip foundations built on the same sand, Oda et al. (1978) demonstrated that the bearing capacity for the model with the load perpendicular to the bedding plane may be $34 \%$ higher than one with a load parallel to the bedding plane. The observed difference in strength is apparently attributable to the effect of cross-anisotropy. Similar observations have been further confirmed by many laboratory tests (Miura and Toki 1982; Azami et al. 2010; Gao et al. 2010). Meanwhile, the undrained shear strength and cyclic liquefaction resistance of sand, which are of great concern in earthquake engineering design, are also found to be strongly dependent on the degree of fabric anisotropy and the relative

\footnotetext{
${ }^{1}$ Lecturer, School of Engineering, Rankine Building, Univ. of Glasgow, Glasgow G12 8LT, U.K. (corresponding author). E-mail: Zhiwei.gao@ glasgow.ac.uk

${ }^{2}$ Associate Professor, Dept. of Civil and Environmental Engineering, Hong Kong Univ. of Science and Technology, Hong Kong.

Note. This manuscript was submitted on April 16, 2014; approved on November 21, 2014; published online on April 23, 2015. Discussion period open until September 23, 2015; separate discussions must be submitted for individual papers. This paper is part of the Journal of Engineering Mechanics, (C) ASCE, ISSN 0733-9399/04015017(15)/\$25.00.
}

orientation between the loading direction and material fabric (Miura and Toki 1982, 1984; Yoshimine et al. 1998; Uthayakumar and Vaid 1998; Oda et al. 2001; Sze and Yang 2014). For instance, Miura and Toki (1982) and Sze and Yang (2014) found that sand deposits with a higher degree of anisotropy (bedding plane is horizontal) show higher undrained shear strength in monotonic triaxial compression tests but lower liquefaction resistance in undrained cyclic triaxial tests. This is mainly because sand samples that are more anisotropic show more contractive responses in the triaxial extension side in cyclic loading. For the same anisotropic sand deposit tested with horizontal and vertical deposition plane orientations, the sample with the horizontal deposition plane has higher undrained shear strength in monotonic triaxial compression tests but lower liquefaction resistance in undrained cyclic triaxial tests, which is also owes to the fact that it shows more contractive response in the triaxial extension side during cyclic loading (Miura and Toki 1984; Oda et al. 2001; Sze and Yang 2014).

To characterize the fabric effect on sand behavior, many theoretical attempts have been made during the past few decades. For example, various constitutive models have been developed to describe the effect of inherent anisotropy on sand responses (e.g., Pietruszczak 1999; Li and Dafalias 2002; Dafalias et al. 2004; Yin et al. 2010). These models are shown to be able to characterize the stress-strain and strength behavior of sand under certain loading conditions with varied degree of satisfaction. However, the assumption of a constant fabric during loading in these models may not be consistent with experimental and numerical observations where sand fabric has been found to change appreciably during loading in order to accommodate the applied stress in an optimum manner (Oda et al. 2001; Cui and O'Sullivan 2006; Li and Li 2009; Li and Dafalias 2012; Guo and Zhao 2013; Zhao and Guo 2013). The evolution of sand fabric, if not properly accounted for, may result in some important features of sand behavior unable to be well captured. Typical examples include the 
noncoaxiality between the principle axes of plastic strain increment and stress (Gao et al. 2014) and the uniqueness of the critical state line ( $\mathrm{Li}$ and Dafalias 2012). There have been attempts to account for the change of anisotropy through incorporating rotational hardening (Sekiguchi and Ohta 1977; Gajo and Muir Wood 2001; Oka et al. 1999) and/or kinematic hardening (Wang et al. 1990; Li 2002). However, as shown by Kaliakin (2003), these techniques consider the loading history only, and are generally unable to adequately account for the influence of fabric and its evolution associated with the physical structure of sand. Proper fabric tensor(s) characterizing the internal structure of sand have to be proposed and incorporated into a constitutive model to render physically realistic and mathematically robust modeling of sand behavior (Oda and Nakayama 1989; Wan and Guo 2001; Dafalias and Manzari 2004; Li and Dafalias 2004; Bauer et al. 2004; Gao et al. 2010). In this regard, Wan and Guo (2001), Li and Dafalias (2012) and Gao et al. (2014) were among the first to develop advanced constitutive models for sand with proper consideration of fabric evolution. These studies are however limited to the description of sand behavior in monotonic shear with fixed principal stress directions. The behavior of sand in relation to fabric and fabric evolution under other general loading conditions (such as constant stress ratio compression and cyclic loading), which is of apparent importance for engineering practice, remains inadequately addressed.

The main objective of this work is to present a comprehensive bounding surface model to describe the fabric effect on sand behavior in both monotonic and cyclic loading based on the recent work by Gao et al. (2014) and the anisotropic critical state theory (Li and Dafalias 2012). An evolving bounding surface and a yield cap are employed to model sand response in constant mean stress shear and constant stress ratio compression, respectively. An anisotropic variable defined by a joint invariant of the fabric tensor and loading direction tensor is conveniently employed to characterize the fabric effect on plastic hardening, plastic flow, and dilatancy of sand in constant mean stress shear. Fabric evolution in both constant mean stress shear and constant stress ratio compression will be considered.

\section{Bounding Surface $\bar{f}_{1}$ and Yield Cap $f_{2}$}

The proposed model is based on the bounding surface concept originally described by Wang et al. (1990) and Li (2002) and the double hardening concept (Vermeer 1978), with further adaptation to be consistent with the anisotropic critical state theory recently developed by Li and Dafalias (2012) and materialized by Gao et al. (2014). Experimental observations show that there is appreciable plastic strain accumulation in sand during cyclic shear (Oda et al. 2001; Kiyota et al. 2008; Chiaro et al. 2009); hence, the authors employ a cone-shaped bounding surface $\bar{f}_{1}$ to describe sand behavior under such loading conditions (Fig. 1). Since the sand behavior is found to be nearly elastic in constant stress ratio unloading and reloading (Pestana and Whittle 1995; Taiebat and Dafalias 2008; Northcutt and Wijewickreme 2013), a yield cap $f_{2}$ perpendicular to the mean stress axis is employed to model sand behavior under such loading conditions (Fig. 1). As a notation convention, all the quantities evaluated on the bounding surface $\bar{f}_{1}$ are distinguished by a superposed bar.

The bounding surface $\bar{f}_{1}$ is expressed as ( $\left.\mathrm{Li} 2002\right)$

$$
\bar{f}_{1}=\bar{R} / g(\bar{\theta})-\bar{H}_{1}=0
$$

where $\bar{R}=\sqrt{3 / 2 \bar{r}_{i j} \bar{r}_{i j}}$ with $\bar{r}_{i j}$ being the image stress ratio tensor of the current stress ratio tensor $r_{i j}=s_{i j} / p=\left(\sigma_{i j}-p \delta_{i j}\right) / p$ (Fig. 2), in which $\sigma_{i j}=$ stress tensor, $s_{i j}=$ deviatoric stress tensor and $\delta_{i j}=$ Kronecker delta $\left(=1\right.$ for $i=j$ and $=0$ for $i \neq j$ ); $\bar{H}_{1}=$ a function of the internal state variables associated with the loading

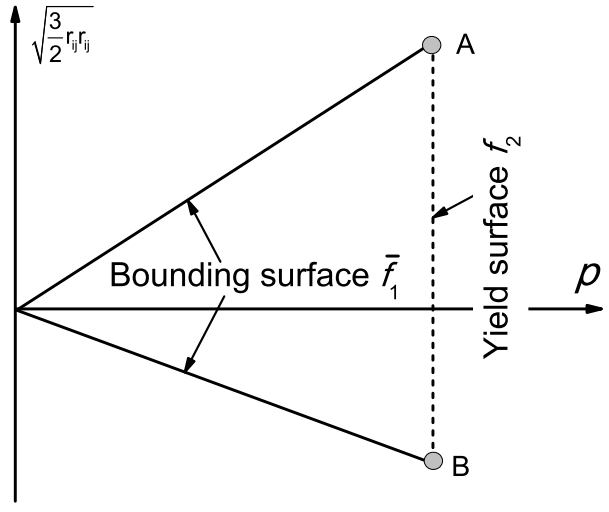

Fig. 1. Bounding surface $\bar{f}_{1}$ and yield cap $f_{2}$

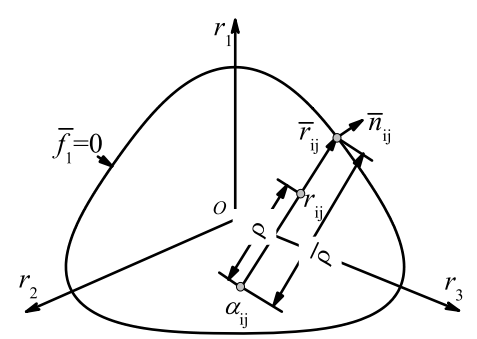

Fig. 2. Mapping rule and the definition of loading direction tensor

history; $g(\bar{\theta})=$ an interpolation function describing the variation of critical state stress ratio with Lode angle $\bar{\theta}$ (Li 2002)

$$
g(\bar{\theta})=\frac{\sqrt{\left(1+c^{2}\right)^{2}+4 c\left(1-c^{2}\right) \sin 3 \bar{\theta}}-\left(1+c^{2}\right)}{2(1-c) \sin 3 \bar{\theta}}
$$

where $c=M_{e} / M_{c}$ with $M_{e}$ and $M_{c}$ denoting the critical state stress ratio in triaxial extension and compression, respectively.

The condition of consistency for the cone, $d \bar{f}_{1}=0$ is expressed as (Li and Dafalias 2002; Li 2002)

$$
d \bar{f}_{1}=p \bar{n}_{i j} d \bar{r}_{i j}-\left\langle L_{1}\right\rangle \bar{K}_{p 1}=p \bar{n}_{i j} d r_{i j}-\left\langle L_{1}\right\rangle K_{p 1}=0
$$

where $\bar{n}_{i j}\left[=\frac{\partial \bar{f}_{1} / \partial \bar{r}_{i j}-\left(\partial \bar{f}_{1} / \partial \bar{r}_{m n}\right) \delta_{m n} \delta_{i j} / 3}{\left\|\partial \bar{f}_{1} / \partial \bar{r}_{i j}-\left(\partial \bar{f}_{1} / \partial \bar{r}_{m n}\right) \delta_{m n} \delta_{i j} / 3\right\|}\right]$ is the deviatoric unit loading direction tensor defined as the norm to $\bar{f}_{1}$ at the image stress ratio point $\bar{r}_{i j}$ (Fig. 2), $\bar{K}_{p 1}$ and $K_{p 1}=$ plastic moduli for the reference and current stress state, respectively; $L_{1}=$ loading index for constant mean stress shear; and \langle\rangle$=$ Macauley brackets such that $\left\langle L_{1}\right\rangle=L_{1}$ for $L_{1}>0$ and $\left\langle L_{1}\right\rangle=0$ for $L_{1} \leq 0$. Eq. (3) indicates the size of the bounding surface $\bar{f}_{1}$ (denoted by $\bar{H}_{1}$ ) increases and decreases when $\bar{K}_{p 1}$ is greater and less than 0 , respectively (Li and Dafalias 2002; Li 2002). An explicit expression of $\bar{H}_{1}$ is not necessarily needed but the evolution of $\bar{H}_{1}$ is provided in Appendix II.

The image stress ratio tensor $\bar{r}_{i j}$ is obtained by the radial mapping rule shown in Fig. 2. In the virgin loading, the projection center $\alpha_{i j}$ is located at the origin of the deviatoric stress ratio space $O$. If $L_{1}$ changes from being positive to negative, $\alpha_{i j}$ will be relocated to the current stress ratio point in the following step (Fig. 3). Therefore, there will be a sudden change in the direction of $\bar{n}_{i j}$ when the projection center $\alpha_{i j}$ is relocated. More detailed discussion on this can be found in Li (2002). In Fig. 3, $\bar{\rho}$ and $\rho=$ the distances of the image and current stress ratio point, respectively, from the projection center $\alpha_{i j}$. Notice that the relocation of the 


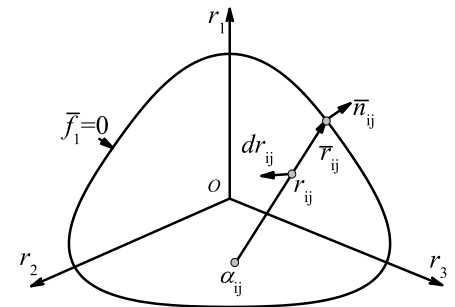

(a)

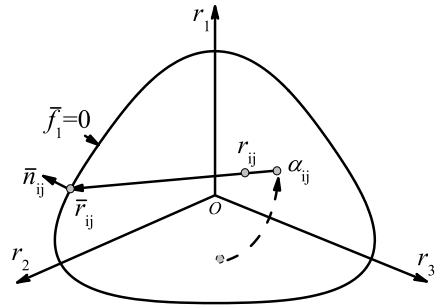

(b)
Fig. 3. Illustration for the relocation of the projection center: (a) current step with negative $L_{1}$; (b) the following step with relocated projection center

projection center in a small unloading and reloading cycle can cause the "overshoot" problem for this model (E-Kan and Taiebat 2014).

The cap yield surface is expressed as ( $\mathrm{Li}$ 2002)

$$
f_{2}=p-H_{2}=0
$$

where $H_{2}=$ location of the flat cap at the mean stress axis. Its initial value is equal to the maximum mean stress to which the sample has been subjected. The condition of consistency for this cap is (Li 2002)

$$
d f_{2}=d p-\left\langle L_{2}\right\rangle K_{p 2}=0
$$

where $L_{2}=$ loading index for constant stress ratio compression and $K_{p 2}=$ plastic modulus for the yield cap. At the initial state, there is a preexisting yield cap $f_{2}$, the location of which is defined by the initial $H_{2}$, which = maximum mean stress the sand sample to which the sample has been subjected. For virgin consolidation before shearing, $\mathrm{H}_{2}$ is just the maximum consolidation pressure. When the current stress state lies on $f_{2}$ and $p$ increases, $H_{2}$ will increase as $K_{p 2}$ is always $\geq 0$ (formulation for $K_{p 2}$ will be shown in the subsequent sections).

Following Gao et al. (2014) and Gao and Zhao (2013), a fabric dependent flow rule expressed as below is employed for constant mean stress shear

$d e_{i j}^{p 1}=\left\langle L_{1}\right\rangle \bar{m}_{i j}, \quad$ with $\quad \bar{m}_{i j}=\frac{\partial \bar{g} / \partial \bar{r}_{i j}-\left(\partial \bar{g} / \partial \bar{r}_{m n}\right) \delta_{m n} \delta_{i j} / 3}{\left\|\partial \bar{g} / \partial \bar{r}_{i j}-\left(\partial \bar{g} / \partial \bar{r}_{m n}\right) \delta_{m n} \delta_{i j} / 3\right\|}$

where $d e_{i j}^{p 1}=$ plastic deviatoric strain increment associated with the loading index $L_{1}$. The plastic potential function $\bar{g}$ is expressed in terms of the fabric tensor $F_{i j}, \bar{r}_{i j}$ and $\bar{n}_{i j}$ as below

$$
\bar{g}=\bar{R} / g(\bar{\theta})-\bar{H}_{g} e^{-k(\bar{A}-1)^{2}}=0
$$

where $k=$ a positive model parameter with default value of 0.03 ; $\bar{A}=$ an anisotropic variable expressed as a joint invariant of $F_{i j}$ and $\bar{n}_{i j}$ (the definition of $\bar{A}$ will be shown in the following section); and $\bar{H}_{g}$ should be adjusted to make $\bar{g}=0$ based on current $\bar{r}_{i j}$ and $F_{i j}$. The plastic potential expressed by Eq. (7), which borrowed the same expression used by Gao et al. (2014), has been based on a micromechanical consideration that the shear resistance of sand is jointly contributed by interparticle friction (denoted by $\bar{H}_{g}$ ) and fabric anisotropy (denoted by $\bar{A}$ ) (see also Nemat-Nasser 2000). Notably, the inclusion of fabric anisotropy via the joint invariant $\bar{A}$ in $\bar{g}$ naturally addresses the noncoaxiality between the plastic strain increment and current stress in monotonic shear when the stress and fabric are initially noncoaxial (Gao et al. 2014;
Gao and Zhao 2013). Eq. (6) is a general expression based on Eq. (7). Notice that the surface of $\bar{g}$ can only be visualized in the principal stress space (or the $\pi$-plane) in special cases with fixed relative orientation between the principal axes of $F_{i j}$ and $\bar{n}_{i j}$ (related to $\bar{r}_{i j}$ ) [see Fig. (10) in Gao et al. 2014 for demonstrative examples], since $\bar{g}$ is a general function dependent on $\bar{A}$, which is a joint invariant of $F_{i j}$ and $\bar{n}_{i j}$.

In constant stress ratio compression, the plastic deviatoric strain increment is assumed to align in the same direction of $r_{i j}$ as follows (Li 2002)

$$
d e_{i j}^{p 2}=\left\langle L_{2}\right\rangle l_{i j}, \quad \text { with } \quad l_{i j}=r_{i j} /\left\|r_{i j}\right\|
$$

where $d e_{i j}^{p 2}$ is the plastic shear strain increment associated with the loading index $L_{2}$.

Assuming that the plastic deviatoric and volumetric strain increments $\left(d e_{i j}^{p}\right.$ and $\left.d \varepsilon_{v}^{p}\right)$ can be decomposed into two parts associated with $L_{1}$ and $L_{2}$, respectively, one has

$$
\begin{gathered}
d e_{i j}^{p}=d e_{i j}^{p 1}+d e_{i j}^{p 2}=\left\langle L_{1}\right\rangle \bar{m}_{i j}+\left\langle L_{2}\right\rangle l_{i j} \\
d \varepsilon_{v}^{p}=d \varepsilon_{v}^{p 1}+d \varepsilon_{v}^{p 2}=\sqrt{2 / 3}\left(D_{1} \sqrt{d e_{i j}^{p 1} d e_{i j}^{p 1}}+D_{2} \sqrt{d e_{i j}^{p 2} d e_{i j}^{p 2}}\right) \\
=\sqrt{2 / 3}\left(\left\langle L_{1}\right\rangle D_{1}+\left\langle L_{2}\right\rangle D_{2}\right)
\end{gathered}
$$

where $\quad D_{1}\left(=d \varepsilon_{v}^{p 1} / \sqrt{2 d e_{i j}^{p 1} d e_{i j}^{p 1} / 3}\right) \quad$ and $\quad D_{2}\left(=d \varepsilon_{v}^{p 2} /\right.$ $\left.\sqrt{2 d e_{i j}^{p 2} d e_{i j}^{p 2} / 3}\right)=$ dilatancy relations for constant mean stress shear and constant stress ratio compression, respectively. It should be mentioned that $d \varepsilon_{v}^{p 2} \geq 0$ as $K_{p 2}>0$ and $d \varepsilon_{v}^{p 2}$ occurs only when $p$ increases.

It is instructive to briefly discuss the interaction between the two loading mechanisms. The shear loading mechanism is active as long as $\bar{n}_{i j} d r_{i j}>0$ [Eq. (3)], and the compression loading mechanism is active only when $p=H_{2}$ and $p$ increases. The interaction between the two mechanisms is detailed in Appendix I. An example of undrained cyclic triaxial compression is also shown in Fig. 4. Both mechanisms are active for path A to B and only the shear loading mechanism is active from $\mathrm{O}$ to $\mathrm{A}$ and $\mathrm{B}$ to $\mathrm{C}$.

\section{Anisotropic Variable $\bar{A}$ and Dilatancy State Parameter $\zeta$}

The evolving bounding surface $\bar{f}_{1}$ and the projection center $\alpha_{i j}$ can help to effectively capture the effect of loading history (e.g., cyclic loading) on sand behavior (Wang et al. 1990; Li 2002; Ling and Yang 2006). Meanwhile, a fabric tensor characterizing the internal

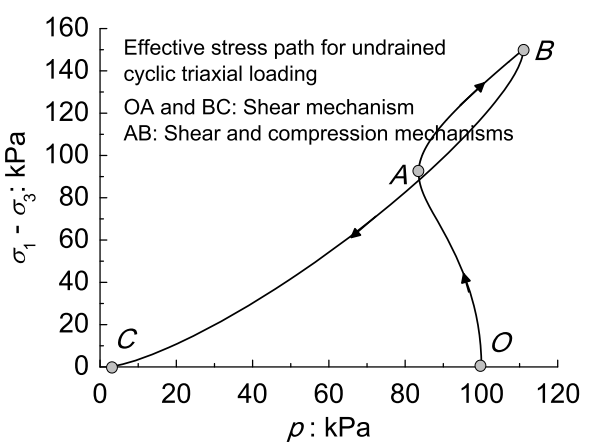

Fig. 4. Illustration for the interaction between the shear and compression loading mechanisms in undrained cyclic loading 
structure of sand also needs to be introduced to account for the fabric effect on sand response ( $\mathrm{Li}$ and Dafalias 2004, 2012; Gao et al. 2014). The current model adopts the void-vector-based deviatoric fabric tensor $F_{i j}$ defined in $\mathrm{Li}$ and $\mathrm{Li}$ (2009). This tensor characterizes the geometric property of the void spaces of a granular assembly and has been shown to be more efficient than other fabric tensors in describing the dilatancy of granular materials $(\mathrm{Li}$ and Li 2009). For an initially crossanisotropic sand sample with the isotropic plane being the $x-y$ plane and the deposition direction aligning with the $z$-axis, the initial $F_{i j}$ can be expressed as

$F_{i j}=\left(\begin{array}{ccc}F_{z} & 0 & 0 \\ 0 & F_{y} & 0 \\ 0 & 0 & F_{x}\end{array}\right)=\sqrt{\frac{2}{3}}\left(\begin{array}{ccc}F_{0} & 0 & 0 \\ 0 & -F_{0} / 2 & 0 \\ 0 & 0 & -F_{0} / 2\end{array}\right)$

where $F_{0}=$ initial degree of anisotropy. For convenience, $F_{i j}$ is normalized in a way that its magnitude $F=\sqrt{F_{i j} F_{i j}}$ is the maximum and unit at the critical state. If one chooses a coordinate system that is not aligned with the deposition direction or the material has been rotated in a fixed coordinate system, a corresponding orthogonal transformation must be carried out.

The following anisotropic variable $\bar{A}$ and dilatancy state parameter $\zeta$ (Li and Dafalias 2012) will be used to characterize the fabric effect on the dilatancy and plastic hardening of sand in constant mean stress shear

$$
\begin{gathered}
\bar{A}=F_{i j} \bar{n}_{i j} \\
\zeta=\psi-e_{A}(\bar{A}-1)=\psi-e_{r}\left[2-(\rho / \bar{\rho})^{x}\right]^{\mu}(\bar{A}-1)
\end{gathered}
$$

where $e_{r}$ and $\mu=$ positive model parameters, $\psi=e-e_{c}=$ state parameter defined by Been and Jefferies (1985) with $e_{c}$ being the critical state void ratio corresponding to the current $p . x=50$ is a default model constant that makes the term $(\rho / \bar{\rho})^{x} \approx 0$ unless $\rho$ is very close to $\bar{\rho}$ (Fig. 3). It can be seen that Eq. (13) guarantees a smooth transition of $e_{A}$ from $e_{r}$ in virgin loading with $\rho / \bar{\rho}=1$ to $2^{\mu} e_{r}$ when the current stress state is inside the bounding surface with $\rho / \bar{\rho}<1$. An $e_{A}$ varying with $\rho / \bar{\rho}$ is used here to gain a better description of sand response in cyclic loading. Consider a case in which the current stress state is inside the bounding surface and $(\rho / \bar{\rho})^{x} \approx 0$, Eq. (13) gives $\zeta=\psi-2^{\mu} e_{r}(\bar{A}-1)$. On the other hand, a constant $e_{A}=e_{r}$ (Li and Dafalias 2012; Gao et al. 2014) will lead to $\zeta^{\prime}=\psi-e_{r}(\bar{A}-1)$, which is typically smaller than $\zeta$ as $\bar{A}-1 \leq 0$. A larger $\zeta$ will render the sand behavior more contractive (see Eq. 18 below) and such consideration is found to offer better description of the sand dilatancy in cyclic loading. Notice that the use of a variable $e_{A}$ will improve model response but the physical significance for such assumption is not clear.

In the present model, the critical state line in the $e-p$ plane is given by (Li and Wang 1998)

$$
e_{c}=e_{\Gamma}-\lambda_{c}\left(p / p_{a}\right)^{\xi}
$$

where $e_{\Gamma}, \lambda_{c}$ and $\xi$ are material constants and $p_{a}(=101 \mathrm{kPa})$ is the atmospheric pressure.

\section{Plastic Modulus and Dilatancy Relation for Constant Mean Stress Shear}

The following plastic modulus is employed in constant mean stress shear

$$
K_{p 1}=\frac{G h}{\bar{R}}\left[M_{c} g(\bar{\theta}) e^{-n \zeta}\left(\frac{\bar{\rho}}{\rho}\right)^{2}-\bar{R}\right]
$$

where $G=$ elastic shear modulus, the expression of which will be shown in the subsequent sections; $n=$ a positive model parameter; and $h=$ a scaling factor for the plastic modulus dependent on the density, $\bar{A}$, and loading history. It can be seen from Eq. (15) that the model gives pure elastic sand response at the onset of loading direction reversal as $K_{p 1}$ is infinite $(\bar{\rho} / \rho=\infty)$, which is supported by experimental observations (Kiyota et al. 2008; Chiaro et al. 2009). When $\bar{\rho}=\rho$ (corresponding to the virgin loading case), $K_{p 1}=\bar{K}_{p 1}=G h / \bar{R}\left[M_{c} g(\bar{\theta}) e^{-n \zeta}-\bar{R}\right]$, which essentially gives a peak stress ratio $\bar{R}$ (or $R$ ) dependent on $\zeta$.

In the present model, the following form of $h$ is used

$$
h=h_{m} h_{c}
$$

where

$$
h_{m}=\left(1-c_{h} e\right) e^{\bar{A}} \quad \text { and } \quad h_{c}=(\rho / \bar{\rho})^{x}+\frac{h_{1}}{(1+F)^{2}}\left[1-(\rho / \bar{\rho})^{x}\right]
$$

where $c_{h}$ and $h_{1}=$ two positive model parameters, $x=50$ renders $h \approx h_{m}$ in the virgin loading with the stress state on $\bar{f}_{1}(\rho / \bar{\rho}=$ $\left.(\rho / \bar{\rho})^{x}=1\right)$, and $h \approx h_{m} h_{1} /(1+F)^{2}$ when the stress state is inside $\bar{f}_{1}\left(\rho / \bar{\rho}<1\right.$ and $\left.(\rho / \bar{\rho})^{x} \approx 0\right)$. The function $h_{m}$ is proposed based on the observations of monotonic sand behavior wherein the shear modulus increases as the void ratio decreases and the anisotropic variable $\bar{A}$ increases (Li and Dafalias 2012). The term $(1+F)^{2}$ is used to render $h_{1}$ to decrease with $F$. This is based on experimental observations that more anisotropic sand samples show a higher rate of positive excess pore pressure accumulation in undrained cyclic loading under otherwise identical conditions (Miura and Toki 1982; Sze and Yang 2014).

The following dilatancy relation in constant mean stress shear is proposed based on the work by Li (2002), Li and Dafalias (2012) and Gao et al. (2014)

$$
D_{1}=\frac{d \varepsilon_{v}^{p 1}}{\sqrt{2 d e_{i j}^{p 1} d e_{i j}^{p 1} / 3}}=\frac{d}{M_{c} g(\bar{\theta})}\left[M_{c} g(\bar{\theta}) e^{m \zeta}\left(\frac{\bar{\rho}}{\rho}\right)-\bar{R}\right]
$$

where

$$
\begin{gathered}
d=d_{1}\left\{(\rho / \bar{\rho})^{x}+\left[1-(\rho / \bar{\rho})^{x}\right] d_{c}\right\} \\
d_{c}=\frac{e^{\omega \int\left\langle-d \varepsilon_{v}^{p 1}\right\rangle}}{1+d_{r} e^{\omega \int\left\langle-d \varepsilon_{v}^{p 1}\right\rangle}}
\end{gathered}
$$

where $m, d_{1}, \omega$ and $d_{r}=$ positive model parameters, $\omega$ is a model constant with default value of 5,000, and $d_{r}=$ relatively small number with default value of 0.1. Eqs. (19) and (20) indicate that $d$ varies smoothly from $d_{1}$ when the stress state is on $\bar{f}_{1}$ in virgin loading to $d_{1} d_{c}$ when the stress state is inside.

The newly introduced $d_{c}$ is used to describe the effect of cyclic loading history on sand dilatancy. It is commonly observed that, during undrained cyclic loading with moderate stress ratio, the rate of excess pore water pressure increases dramatically at the onset of loading direction reversal when the stress state goes above the phase transformation line, which may be attributable to the fact that the highly anisotropic void space system that has developed due to fabric evolution can be extremely unstable when the loading direction changes (Oda et al. 2001; Sazzad and Suzuki 2010; Soroush and Ferdowsi 2011). It can be seen from Eq. (20) that $d_{c}$ will increase dramatically after the occurrence of the first phase transformation $\left(\int\left\langle-d \varepsilon_{v}^{p 1}\right\rangle\right.$ becomes positive). This is because $\omega$ is very big 
and a small increase in $\int\left\langle-d \varepsilon_{v}^{p 1}\right\rangle$ can result in big increase in $e^{\omega \int\left\langle-d \varepsilon_{v}^{p 1}\right\rangle}$ and $d_{c}$ according to Eq. (20). The integral $\int\left\langle-d \varepsilon_{v}^{p 1}\right\rangle$ keeps increasing as long as $d \varepsilon_{v}^{p 1}<0$ and remains unaltered to the value they have reached when the stress reversal takes place above the phase transformation line. The maximum $d_{c}$ is around 10 when $\bar{R} / M_{c} g(\bar{\theta})=1$ and $e^{\omega \int\left\langle-d \varepsilon_{v}^{p 1}\right\rangle}=\infty$. Therefore, the model gives an increasing $D_{1}$ after the first phase transformation. Higher $D_{1}$ leads to a higher rate of excess pore pressure accumulation in undrained cyclic loading. It is worth mentioning that Eq. (20) is essential for getting a better fit of sand behavior in cyclic loading but has its own drawback as one cannot distinguish whether the plastic volumetric strain is caused by the shear or compression mechanism. Indeed, other similar approaches have also been proposed to model the cyclic loading history on sand behavior by employing plastic deformation-dependent dilatancy relation and/or plastic modulus (e.g., Wang et al. 1990; Oka et al. 1999; Li 2002; Ling and Yang 2006; Wang and Xie 2014).

\section{Plastic Modulus and Dilatancy Relation for Constant Stress Ratio Compression}

We propose the following plastic modulus under constant stress ratio loading

$$
K_{p 2}=\sqrt{\frac{2}{3}} d_{2} \frac{M_{c} g(\theta)}{R r_{2}}
$$

where $\theta=$ Lode angle for $r_{i j}, R=\sqrt{3 r_{i j} r_{i j} / 2}$ is the current stress ratio, and $d_{2}=$ a positive model parameter. The expression for $r_{2}$ describes the $e-p$ relation in constant stress ratio compression, which is always greater than zero. The term $\sqrt{2 / 3}$ is added to offer a simpler relation between $d \varepsilon_{v}^{p 2}$ and $d p$ [Eq. (23)], where $d \varepsilon_{v}^{p 2}=$ plastic volumetric strain increment in constant stress ratio compression and $d p=$ increment of mean effective stress. It follows from Eqs. (5), (8), and (20) that $K_{p 2}=\infty$ at $R=0$, and thus, $L_{2}=0$, which indicates that no plastic shear strain occurs in isotropic compression. $K_{p 2}$ becomes finite when $R>0$ and plastic shear strain is produced for constant stress ratio compression with $R>0$. Eq. (21) is proposed to have such features based on the following experimental observations. In isotropic compression with $R=0$, shear strain is not expected for an isotropic sample and negligible amount of plastic shear strain is found for an anisotropic one (Abelev et al. 2007). In a constant stress ratio compression with $R>0$, however, shear strain is always observed for both isotropic and anisotropic samples (McDowell et al. 2002; Northcutt and Wijewickreme 2013).

The dilatancy in constant stress ratio loading is expressed as follows

$$
D_{2}=\frac{d \varepsilon_{v}^{p 2}}{\sqrt{2 d e_{i j}^{p 2} d e_{i j}^{p 2} / 3}}=d_{2} \frac{M_{c} g(\theta)}{R}\left\langle 1-\left[R / M_{c} g(\theta)\right]^{x}\right\rangle
$$

where $x=50$ is a default big number that renders $1-$ $\left[R / M_{c} g(\theta)\right]^{x} \approx 1$ when $R<M_{c} g(\theta)$. The McCauley brackets \langle\rangle are to prevent $D_{2}$ becoming negative at $R>M_{c} g(\theta)$ (plastic volumetric expansion is not expected in constant stress ratio compression) and guarantee zero dilatancy at the critical state (Li 2002). Note that a similar dilatancy relation has also been employed in other models (Wang et al. 1990; Taiebat and Dafalias 2008).

Based on Eqs. (5), (10), (21), and (22), the compressive behavior of sand under constant stress ratio loading $\left[R<M_{c} g(\theta)\right]$ can be obtained as below

$$
d \varepsilon_{v}^{p 2} \approx r_{2} d p
$$

In the present model, the expression for $r_{2}$ is proposed based on Taiebat and Dafalias (2008)

$$
r_{2}=\frac{e}{1+e}\left[\rho_{c}-\frac{\left(p / p_{a}\right)^{1 / 3}}{K_{0}}\right] \frac{1}{p}\left(1-\operatorname{sgn} \delta|\delta|^{\beta}\right)
$$

where $K_{0}=$ a model parameter for the elastic modulus of sand, $\beta=$ a parameter that controls the curvature of the predicted $e-p$ relation in constant stress ratio compression, $\rho_{c}$ is the slope of the limit compression curve (LCC) for isotropic compression in the $\log e-$ $\log p$ space (Pestana and Whittle 1995), and

$$
\delta=1-\frac{p}{p_{b}}\left[1+2\left(\frac{R}{M_{c} g(\theta)}\right)^{2}\right]
$$

where $p_{b}=$ image mean stress on the LCC for isotropic compression corresponding to the current void ratio $e$ (Pestana and Whittle 1995; Taiebat and Dafalias 2008). The expression for the LCC in isotropic compression is $\log e=\rho_{c} \log \left(p_{r} / p\right)$, where $p_{r}=$ means stress corresponding to $e=1$ on the LCC. For more detailed discussion on the derivation of Eqs. (24) and (25), please refer to Pestana and Whittle (1995) and Taiebat and Dafalias (2008).

The same $d_{2}$ is employed to express the plastic modulus in Eq. (21) and the dilatancy relation in Eq. (22), which facilitates the derivation of Eq. (23). However, it does not imply that $d_{2}$ has the same effect on both $d \varepsilon_{v}^{p 2}$ and $d \varepsilon_{q}^{p 2}\left(=\sqrt{2 d e_{i j}^{p 2} d e_{i j}^{p 2} / 3}\right)$ in constant stress ratio compression. Indeed, the relation between $d \varepsilon_{v}^{p 2}$ and $d p$ is uniquely controlled by the expression for $r_{2}$ in Eq. (24) while the relation between $d \varepsilon_{q}^{p 2}$ and $d p$ is dependent on $d_{2}$. Nevertheless it should be noted that the model gives a constant $K_{0}$ value only when $p$ is large enough to cause particle crushing, and hence may not be particularly effective in predicting the $K_{0}$ behavior in sand.

\section{Fabric Evolution}

It remains a challenging task to measure the fabric and its evolution of sand in the laboratory. Knowledge of fabric evolution of granular materials has been mainly based on micromechanics-based investigations such as distinct element simulations (Li and Li 2009; Guo and Zhao 2013; Zhao and Guo 2013). By neglecting potential fabric change due to pure elastic deformation, the following fabric evolution is assumed in the present model

$$
d F_{i j}=k_{f}\left\{\left(\bar{n}_{i j}-F_{i j}\right) d \varepsilon_{q}^{p 1}+\left[\frac{R}{M_{c} g(\theta)} l_{i j}-F_{i j}\right] d \varepsilon_{v}^{p 2}\right\}
$$

where $k_{f}=$ a model parameter describing the rate of fabric evolution with plastic strain increment associated with $d \varepsilon_{q}^{p 1}\left(=\sqrt{2 d e_{i j}^{p 1} d e_{i j}^{p 1} / 3}\right)$ and $d \varepsilon_{v}^{p 2}$. Note that the same $k_{f}$ is used for $d \varepsilon_{v}^{p 2}$ and $d \varepsilon_{v}^{p 1}$ for simplicity. A better and more reasonable description of fabric evolution under general loading conditions can be obtained if different values of $k_{f}$ are used for the two loading mechanisms. It is worth mentioning that $d \varepsilon_{q}^{p 2}$ cannot be used in Eq. (26) as $d \varepsilon_{q}^{p 2}=0$ in isotropic compression for the present model. According to Abelev et al. (2007), fabric evolution does occur under such loading conditions when the sample is initially anisotropic. Eq. (26) indicates that $F_{i j}$ will eventually become codirectional with $\bar{n}_{i j}$ and reach a constant magnitude of $F=1$ when $\varepsilon_{q}^{p 1}\left(=\int d \varepsilon_{q}^{p 1}\right)$ is sufficiently large, which complies with the anisotropic critical state theory (Li and Dafalias 2012). In a pure constant stress ratio compression, Eq. (26) will not lead $F_{i j}$ to 

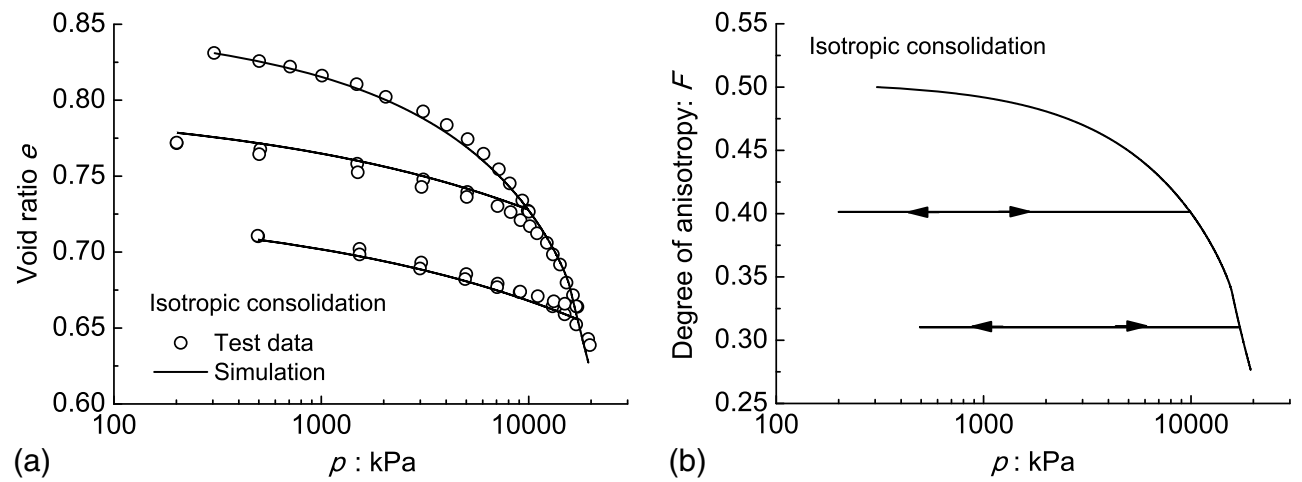

Fig. 5. (a) Comparison between the model simulation and test results for the $e$ - $p$ relation of Toyoura sand in isotropic compression (test data from Miura et al. 1984); (b) simulated fabric evolution

critical state but give a material fabric that is codirectional with $l_{i j}$ and has a constant magnitude $F=R / M_{c} g(\theta)$ when $\varepsilon_{v}^{p 2}\left(=\int d \varepsilon_{v}^{p 2}\right)$ is large enough. This assumption is reasonable as the sample will not reach the critical state if it is subjected to constant stress ratio compression only. The fabric evolution law [Eq. (26)] can also be expressed as below based on Eqs. (9) and (10),

$$
d F_{i j}=\sqrt{\frac{2}{3}} k_{f}\left\{\left\langle L_{1}\right\rangle\left(\bar{n}_{i j}-F_{i j}\right)+\left\langle L_{2}\right\rangle D_{2}\left[\frac{R}{M_{c} g(\theta)} l_{i j}-F_{i j}\right]\right\}
$$

Fig. 5 shows the simulated $e-p$ relation and fabric evolution in isotropic compression. It can be seen that $F$ decreases with the accumulation of plastic volumetric strain [Fig. 5(b)], which is in agreement with the experimental observations by Abelev et al (2007). In the unloading and reloading cycles, the model gives pure elastic response [Fig. 5(a)] and the fabric does not evolve [Fig. 5(b)]. Fig. 6 shows the simulated stress-strain relation and fabric evolution in a drained triaxial test with one unloading and reloading cycle. The fabric and stress are initially codirectional and $F_{0}=0.5$. For this case, fabric evolution is dominated by the shear loading mechanism as $d \varepsilon_{q}^{p 1}$ is much bigger than $d \varepsilon_{v}^{p 2}$. At large strain level, the fabric reaches the critical state with a constant magnitude 1 , which complies with the anisotropic critical state theory ( $\mathrm{Li}$ and Dafalias 2012). Noting that the loading direction $\bar{n}_{i j}$ reverses when unloading occurs (Fig. 3), and the fabric $F_{i j}$ will hence adjust itself to become codirectional with the loading direction [Eq. (26)]. Specifically, the major principal component decreases and the minor principal component increases, which makes $F$ decrease [Fig. 6(b)]. At the onset of loading direction reversal, there is a sudden change in
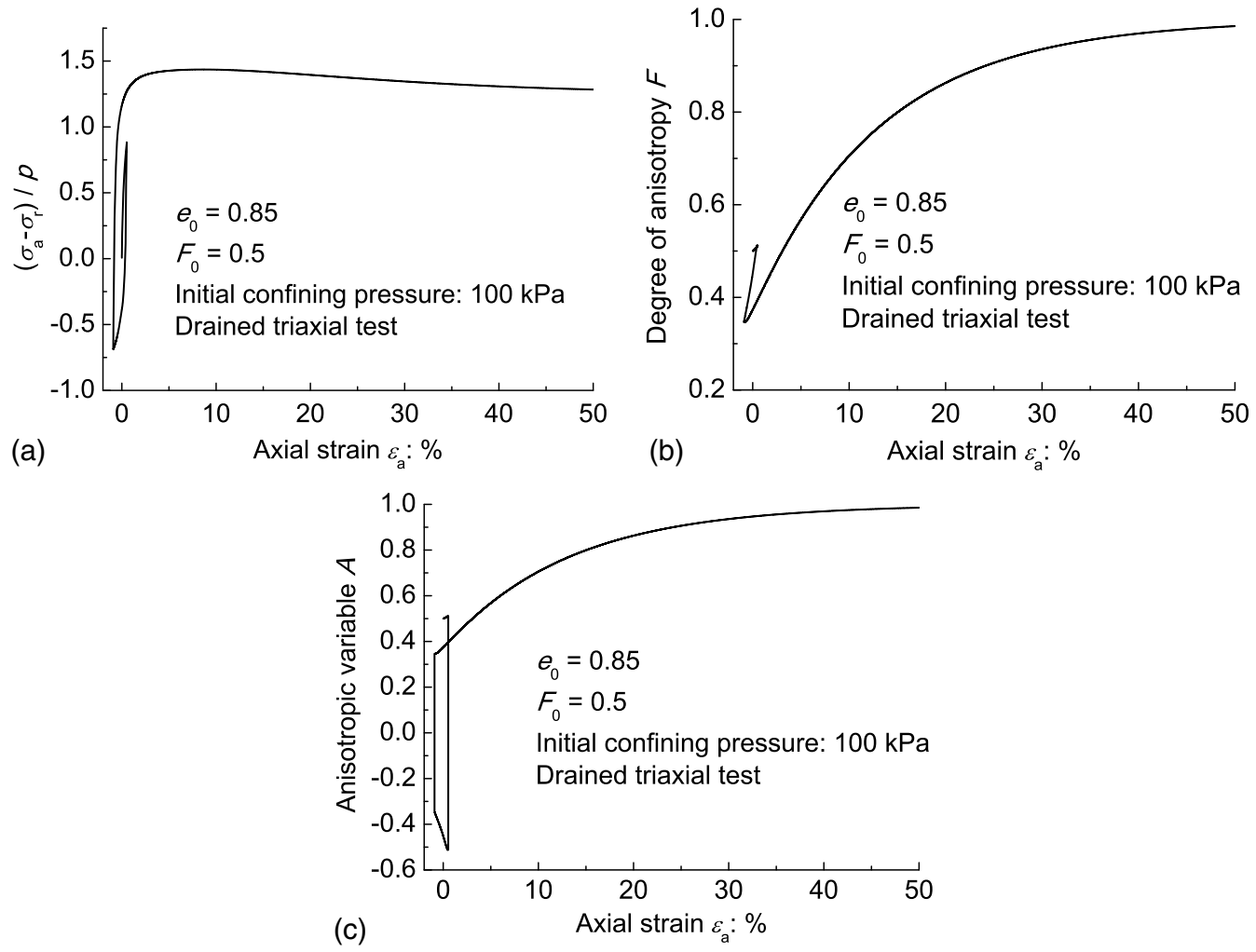

Fig. 6. Model simulation for (a) the stress-strain relation; (b) evolution of F; (c) evolution of $A$ for Toyoura sand in a drained triaxial compression test 
Table 1. Summary of Model Parameters for Toyoura Sand

\begin{tabular}{lccc}
\hline Parameter & Symbol & Value & Typical range \\
\hline Elasticity & $G_{0}$ & 125 & $120-150$ \\
Critical state & $K_{0}$ & 150 & $120-160$ \\
& $M_{c}$ & 1.25 & $1.0-1.6$ \\
& $c$ & 0.75 & $0.75-0.8$ \\
& $e_{\Gamma}$ & 0.934 & $0.9-1.1$ \\
& $\lambda_{c}$ & 0.019 & $0.01-0.03$ \\
Parameters associated with & $\lambda_{c}$ & 0.7 & Around 0.7 \\
constant mean stress shear & $c_{h}$ & 0.90 & $0.8-0.9$ \\
& $e_{r}$ & 0.09 & $0.09-0.11$ \\
& $n$ & 4.0 & $2.0-5.0$ \\
& $d_{1}$ & 0.4 & $0.2-0.6$ \\
& $m$ & 5.3 & $1.0-6.0$ \\
& $h_{1}$ & 7.6 & $3.0-10.0$ \\
Parameters associated with & $\omega$ & 5,000 & Default value \\
constant stress ratio & $\rho_{c}$ & 0.1 & Default value \\
compression & $\rho_{r}$ & 1 & Assumed \\
Fabric evolution & $\beta$ & 0.37 & $0.3-0.4$ \\
Initial anisotropy & $d_{2}$ & $5,500.0$ & $3,000-6,000$ \\
\hline & $d_{f}$ & 1 & $0.1-0.3$ \\
& $F_{0}$ & 0.5 for Figs. 7-10 & Assumed \\
& & & $7.0-8.0$ \\
\hline
\end{tabular}

$A$ [Fig. 6(c)]. The model parameters used in the two simulations above are shown in Table 1. More discussion on the fabric evolution and its relation with sand behavior can be found in Gao et al. (2014).

\section{Elastic Stress-Strain Relations}

Hypoelastic stress-strain relations are used in this model. For the elastic shear modulus $G$, the equation by Richard et al. (1970) is adopted, which is a function of $p$ and $e$ expressed as
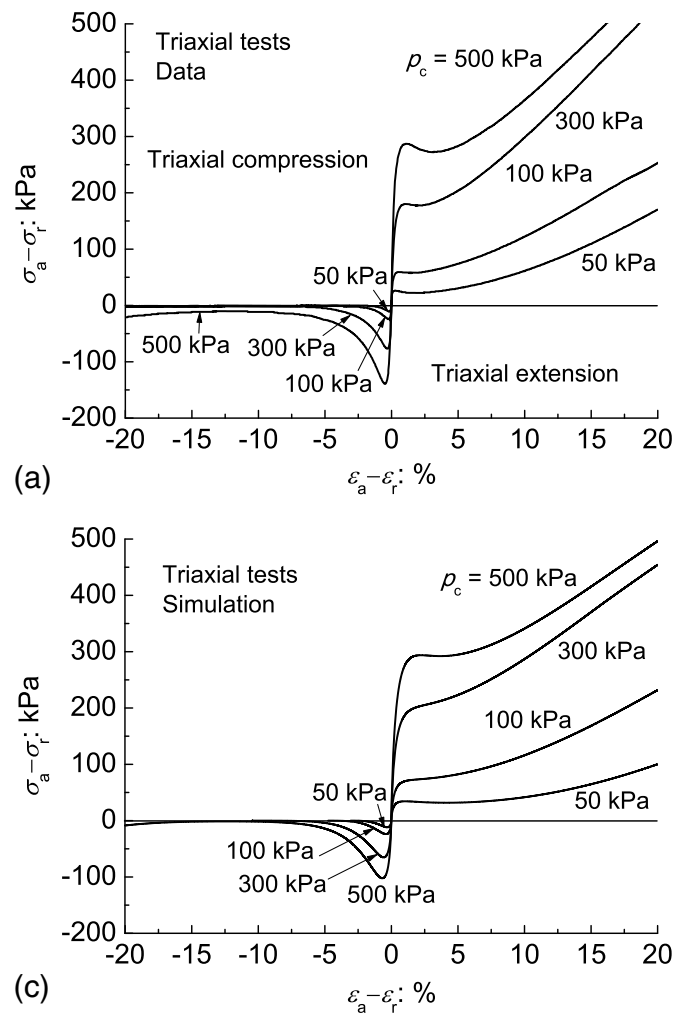

$$
G=G_{0} \frac{(2.97-e)^{2}}{1+e} \sqrt{p p_{a}}
$$

where $G_{0}=$ a model parameter.

Following Taiebat and Dafalias (2008) and Pestana and Whittle (1995), the elastic bulk modulus $K$ expressed below is used for the present model

$$
K=K_{0} p_{a} \frac{1+e}{e}\left(\frac{p}{p_{a}}\right)^{2 / 3}
$$

Note that Eq. (29) has been used to derive Eq. (24) in Taiebat and Dafalias (2008). One may also use $K=2 G(1+\nu) / 3(1-2 \nu)$ to obtain $K$ based on Eq. (28) where $\nu=$ Poisson's ratio. However, the behavior of sand in isotropic compression may not be well captured.

\section{Determination of Model Parameters}

The initial degree of anisotropy $F_{0}$ needs to be determined before the model parameters. Since it remains difficult to measure the fabric of sand using conventional laboratory tests and other in situ test techniques, $F_{0}=0.5$ is simply assumed for Toyoura sand prepared by dry deposition in several layers (Yoshimine et al. 1998) and air pluviation (Kiyota et al. 2008; Chiaro et al. 2009) in this study (Figs. 7-10). Different $F_{0}$ is used for Toyoura sand prepared by other methods (see the text below). A feasible way to determine $F_{0}$ may be based on the anisotropic elastic stiffness tensor of sand, which can be expressed as a function of the fabric tensor (Cowin 1985; Lashkari 2010). Since the initial stress state is isotropic for all the simulations here, the initial $\bar{H}_{1}=0$ and the initial $H_{2}=$ mean effective stress after consolidation.
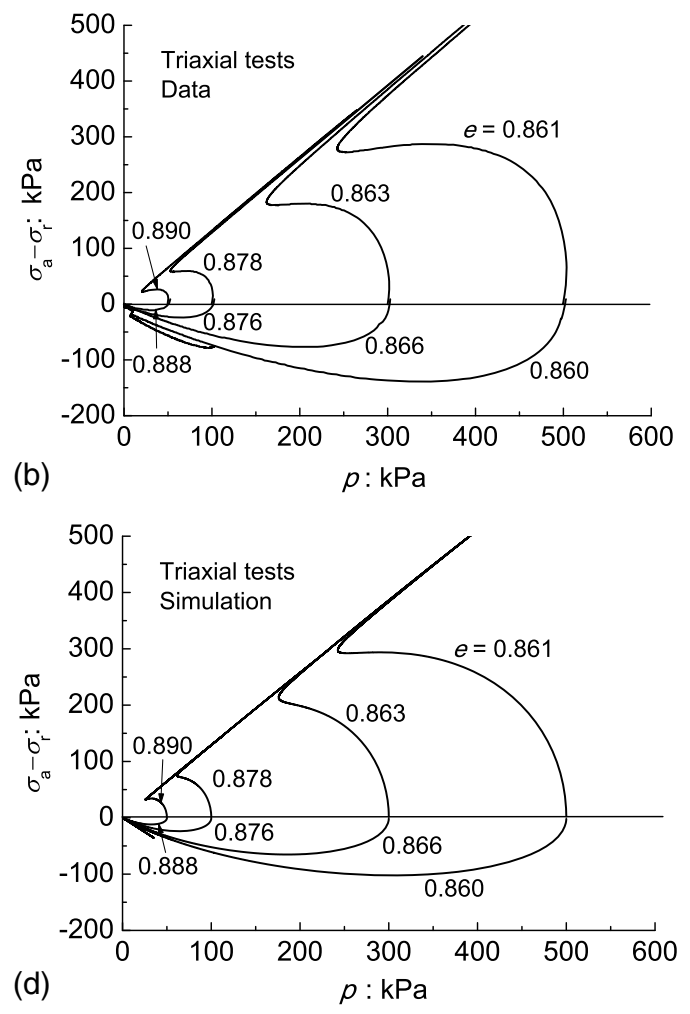

Fig. 7. Comparison between the observed and model simulated behavior of Toyoura sand in undrained triaxial tests (data from Yoshimine et al. 1998) 

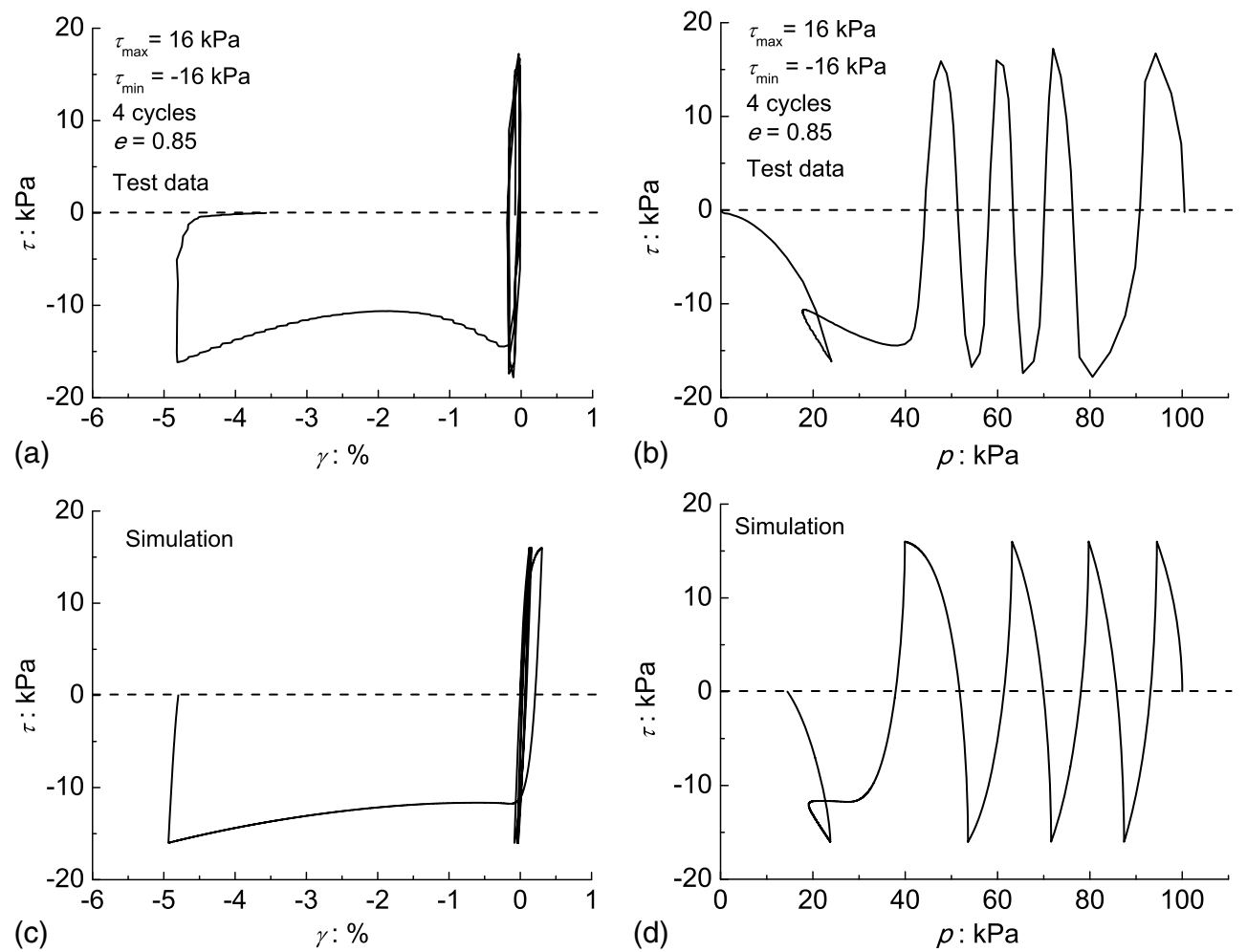

Fig. 8. Comparison between the model simulations and test results for the behavior of Toyoura sand in undrained cyclic simple shear test (data from Chiaro et al. 2009)
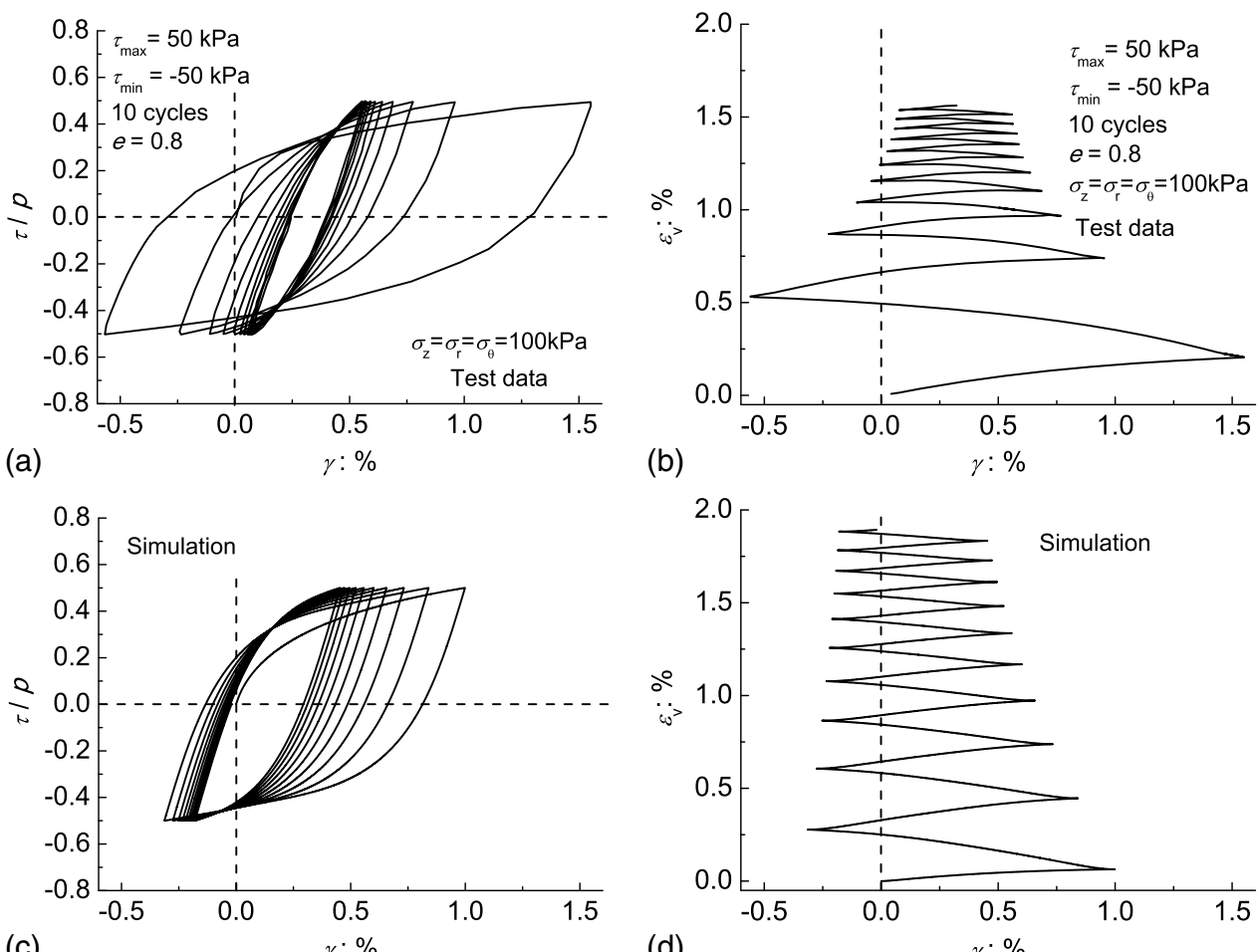

(c)

(d)

$\gamma: \%$

Fig. 9. Comparison between the model simulations and test results for the behavior of Toyoura sand with $e=0.8$ in drained cyclic simple shear test (data from Wahyudi et al. 2010) 

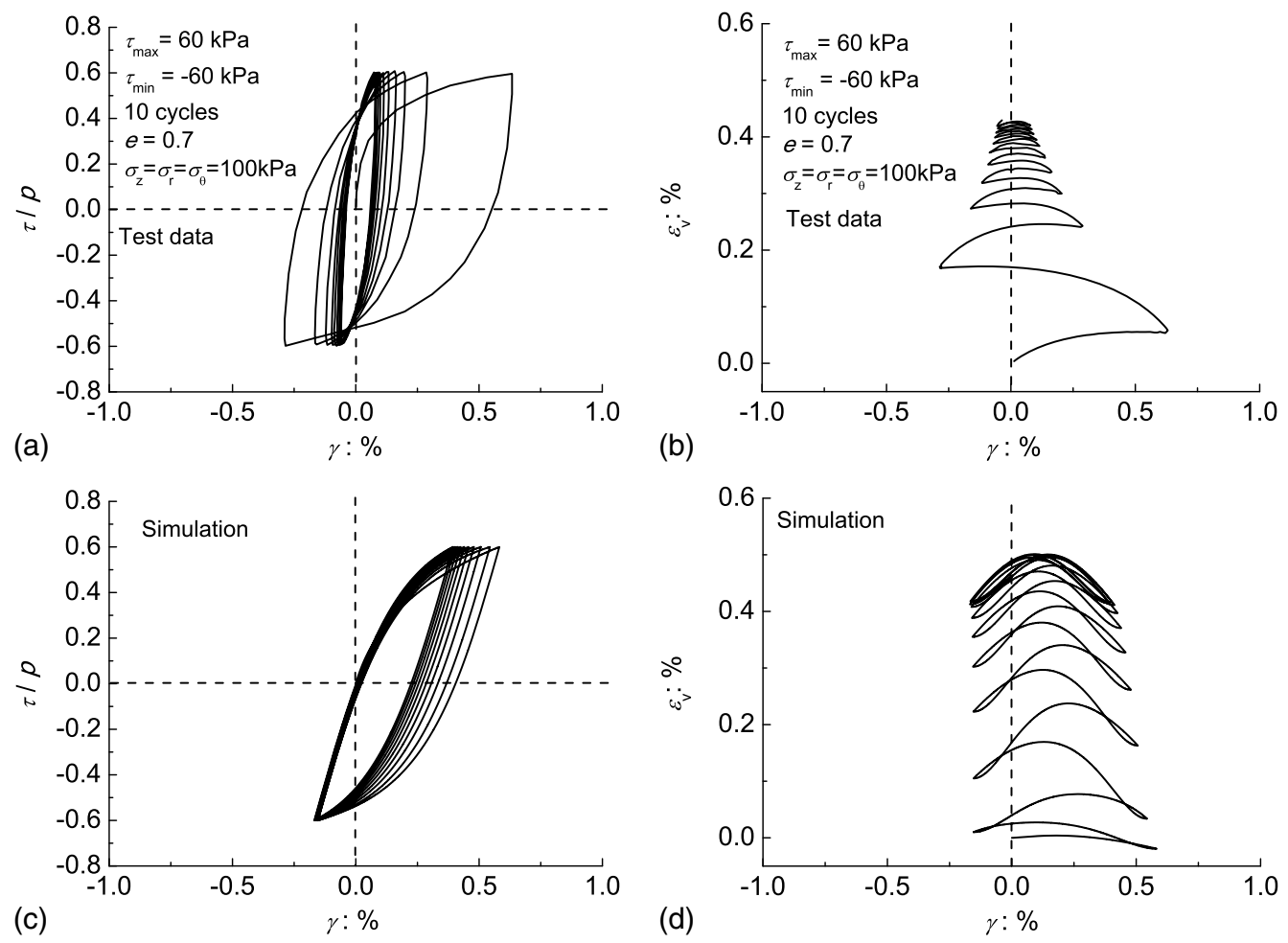

Fig. 10. Comparison between the model simulations and test results for the behavior of Toyoura sand with $e=0.7$ in drained cyclic simple shear test (data from De Silva 2008)

There are six groups of model parameters, and their values for Toyoura sand are shown in Table 1 . The calibration method will be discussed in the following:

1. Elastic parameters. The elastic parameter $K_{0}$ can be determined according to the $e-p$ relation in constant stress ratio unloading [e.g., the unloading curve in isotropic consolidation tests shown in Fig. 5(a)]. The parameter $G_{0}$ can be determined based on the stress-strain relations at the very beginning of the triaxial tests (Taiebat and Dafalias 2008). Note that the maximum pressure level in Fig. 5 can cause particle crushing but this model does not consider the effect of particle crushing on sand behavior.

2. Critical state parameters. The critical state parameters can be obtained directly from the critical state stress ratio in triaxial compression and extension (for $M_{c}$ and $c$ ) and the location of the critical state line in the $e-p$ plane (for $e_{\Gamma}, \lambda_{c}$ and $\xi$ ).

3. Parameters relevant to constant mean stress shear. There are nine parameters associated with constant mean stress shear. They can be determined based on shear-dominated tests such as monotonic and cyclic triaxial and simple shear tests. The parameter $c_{h}$ varies in a small range and only fine tuning is needed for different sands to capture the effect of the void ratio on plastic hardening of sand in monotonic loading (Gao et al. 2014). The parameter $e_{r}$ describes the effect of fabric anisotropy and loading direction on dilatancy and plastic hardening of sand in shear-dominated monotonic loading. It can thus be determined by fitting the test results in triaxial extension. It is also found that the variation of $e_{r}$ is small for different sands (Gao et al. 2014). The parameters $n, d_{1}$, and $m$ can be determined by trial and error to fit the monotonic triaxial compression tests. It is found that these parameters $\left(c_{h}, e_{r}, n, d_{1}\right.$, and $m$ ) are closely related to the particle constitution of sand (gradation, maximum, and minimum void ratio) (Gao et al. 2014) and their typical ranges are shown in Table $1 . \mu=1$ is assumed in this paper. The parameter $h_{1}$ controls the value of plastic modulus $K_{p 1}$ in cyclic loading and thence controls the rate of excess pore pressure accumulation in undrained cyclic loading. It can be determined by best fitting the effective stress paths in undrained cyclic loading. A default value of 5,000 and 0.1 can be sued for $\omega$ and $d_{r}$, respectively.

4. Parameters relevant to constant stress ratio compression. The parameters $\rho_{c}$ and $p_{r}$ can be directly obtained based on the location of the LCC for isotropic compression in the $\log e-$ $\log p$ space and $\beta$ can be determined by best fitting the isotropic/one-dimensional compression curve in the $e-p$ plane [Fig. 4(a)]. Since there is no test data available for dilatancy of Toyoura sand in constant stress ratio compression, parameter $d_{2}$ is assumed to be 1 here.

5. Fabric evolution parameter. While it is still not possible to measure the fabric evolution in laboratory tests, $k_{f}$ cannot be directly obtained. It is found that the predicted fabric evolution with $k_{f}=7.0-8.0$ is in qualitative agreement with the distinct element simulations, especially when static liquefaction occurs (Gao et al. 2014). Therefore, $k_{f}=7.35$ is used in this study and can be treated as a constant for other sands with particle constitution similar to that of Toyoura sand.

\section{Model Simulations for Sand Behavior in Monotonic Loading}

Fig. 7 shows the model simulations of undrained tests of drydeposited Toyoura sand in monotonic triaxial compression and extension in comparison with laboratory test results. The test setup and loading conditions have been discussed in Yoshimine et al. (1998). In Fig. 7, $\sigma_{a}, \sigma_{r}, \varepsilon_{a}$, and $\varepsilon_{r}$ denote the axial effective stress (in the vertical direction), radial effective stress, axial strain, and radial strain, respectively. It can be observed that the model captures the effect of confining pressure, fabric anisotropy, and density 
on monotonic sand behavior reasonably well. Since the current model has been based on that in Gao et al. (2014), which was proposed for monotonic shear, more of its predictive capacity for the monotonic loading case can be referred to that early study.

\section{Model Simulations for Sand Behavior in Cyclic Simple Shear}

This section will present the model simulations for sand behavior in both drained and undrained cyclic simple shear. The test results for Toyoura sand prepared by air pluviation (Kiyota et al. 2008; Chiaro et al. 2009) will be used. Since this sample preparation method is similar to the dry deposition method used by Yoshimine et al. (1998), $F_{0}=0.5$ is also used for these sand samples.

Fig. 8 compares the model simulations against test data for Toyoura sand in undrained cyclic simple shear tests. For the test shown here, the sample was first isotropically consolidated to $p=$ $100 \mathrm{kPa}$ and cyclic undrained simple shear was then applied with constant amplitude of shear stress $\tau$ (Chiaro et al. 2009). Evidently, the model gives good predictions for the effective stress path and shear stress-strain relation. In Figs. $8-10, \tau_{\max }$ and $\tau_{\min }=$ maximum and minimum shear stresses in each cycle, respectively, and $\gamma=$ shear strain.

Figs. 9 and 10 show the comparison between the model simulations and test results for drained cyclic simple shear behavior of Toyoura sand. The samples were first isotropically consolidated to $100 \mathrm{kPa}$ and constant amplitude of shear stress (50 kPa for Fig. 9 and $60 \mathrm{kPa}$ for Fig. 10) was then applied by keeping all the normal stress components constant. Figs. 9 and 10 show the circumferential stress. The model captures the main characteristics of sand behavior in cyclic drained loading but the simulations are not quite accurate. The model simulations can be improved in the following aspects. First, the double amplitude cyclic strain decreases as the number of cycles increases, but the rate is smaller than in the observations. Better model performance is expected if the $h_{c}$ of Eqs. (16) and (17) is also assumed to be dependent on the plastic strain accumulation during cyclic loading, which renders the plastic modulus increase with the accumulation of plastic volumetric or shear strain (see also Ling et al. 2006 and Wang and Xie 2014). Secondly, the model gives stiffer shear modulus for both tests. The model performance can be improved by better expression for $h_{m}$ which can give better description of sand stiffness at relatively low shear strain level.

\section{Model Description for the Effect of Fabric Anisotropy on Cyclic Sand Response}

Figs. 11( $\mathrm{a}$ and $\mathrm{b}$ ) show the undrained cyclic triaxial test results on Toyoura sand prepared by two different methods (Miura and Toki 1982). The monotonic triaxial test results indicate that the sample prepared by the wet rodding method is approximately isotropic (Miura and Toki 1982), and thus $F_{0}=0$ is used in the simulations [Fig. 11(c)]. The sample prepared by the multiple sieving pluviation method is found to be initially anisotropic (Miura and Toki 1982), and its initial degree of anisotropy is set to be $F_{0}=0.22$ based on best fitting of the effective stress path shown in Fig. 11(b and d). Note that the model parameters listed in Table 1 (except $F_{0}$ ) are used for these two samples.

Although the predicted effective stress path shows relatively large deviation from the measured one for the sample prepared by the multiple sieving pluviation method, the model does give reasonable characterizations of the fabric effect on the sand behavior in cyclic loading, i.e., more isotropic sample shows higher liquefaction resistance in undrained cyclic triaxial tests ( $p$ decreases more slowly with the number of cycles). For example, at the end of the sixth cycle, $p$ for the sample
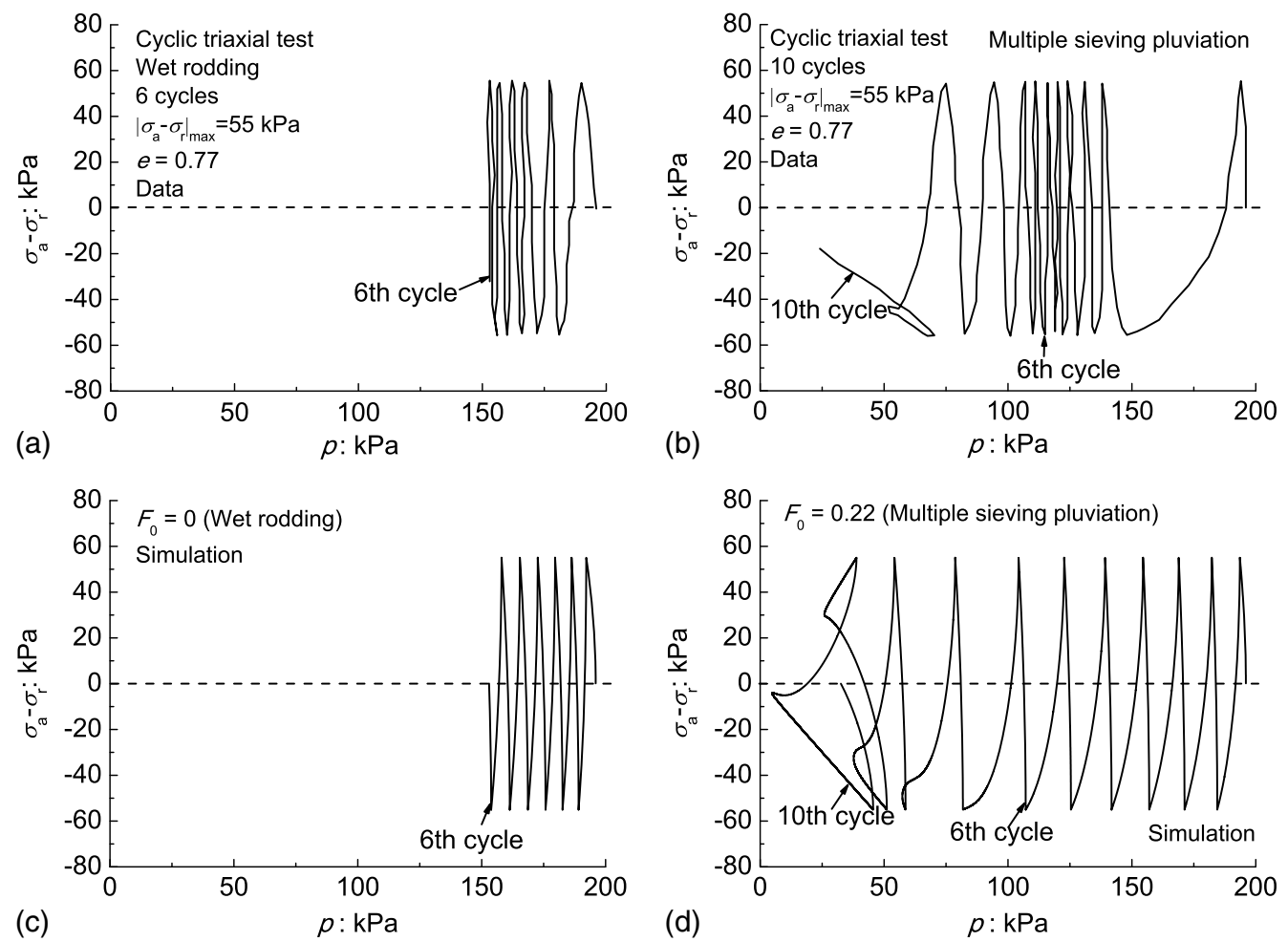

Fig. 11. Model simulations for the effect of initial degree of anisotropy on sand behavior in undrained cyclic triaxial test (test data from Miura and Toki 1982) 

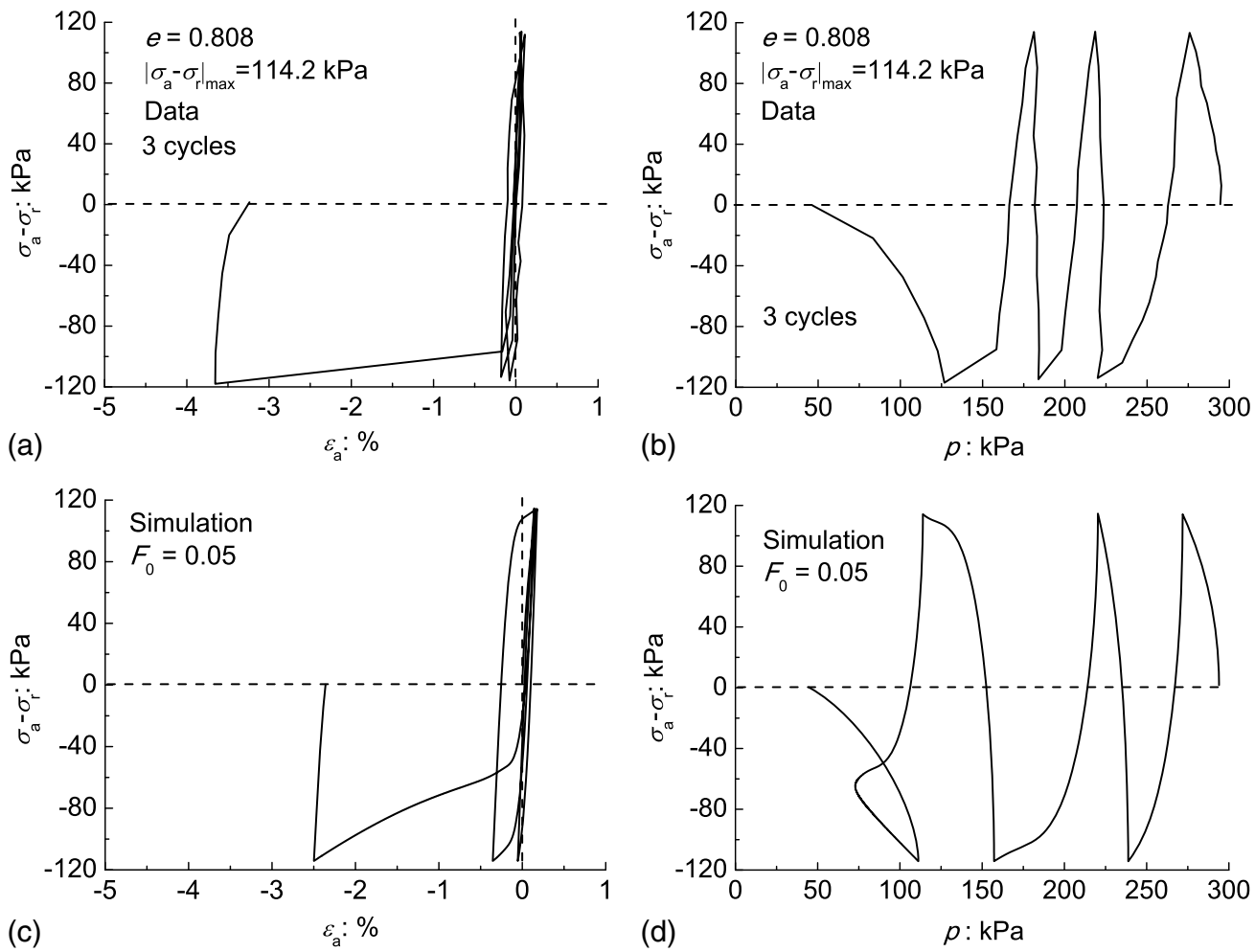

Fig. 12. Comparison between model simulations and test results on the behavior of Toyoura sand in undrained cyclic triaxial test (data from Dafalias and Manzari 2004)

prepared by multiple sieving pluviation is around $110 \mathrm{kPa}$, which is lower than that for the wet-rodded sample $(155 \mathrm{kPa})$ [Figs. 9(a and b)]. Figs. 11(c and d) indicate that the model is capable of capturing this difference. The stress-strain loops are not available and the corresponding simulations are not presented.

Fig. 12 shows the model simulation for the behavior of Toyoura sand in the cyclic triaxial test. The sample was prepared
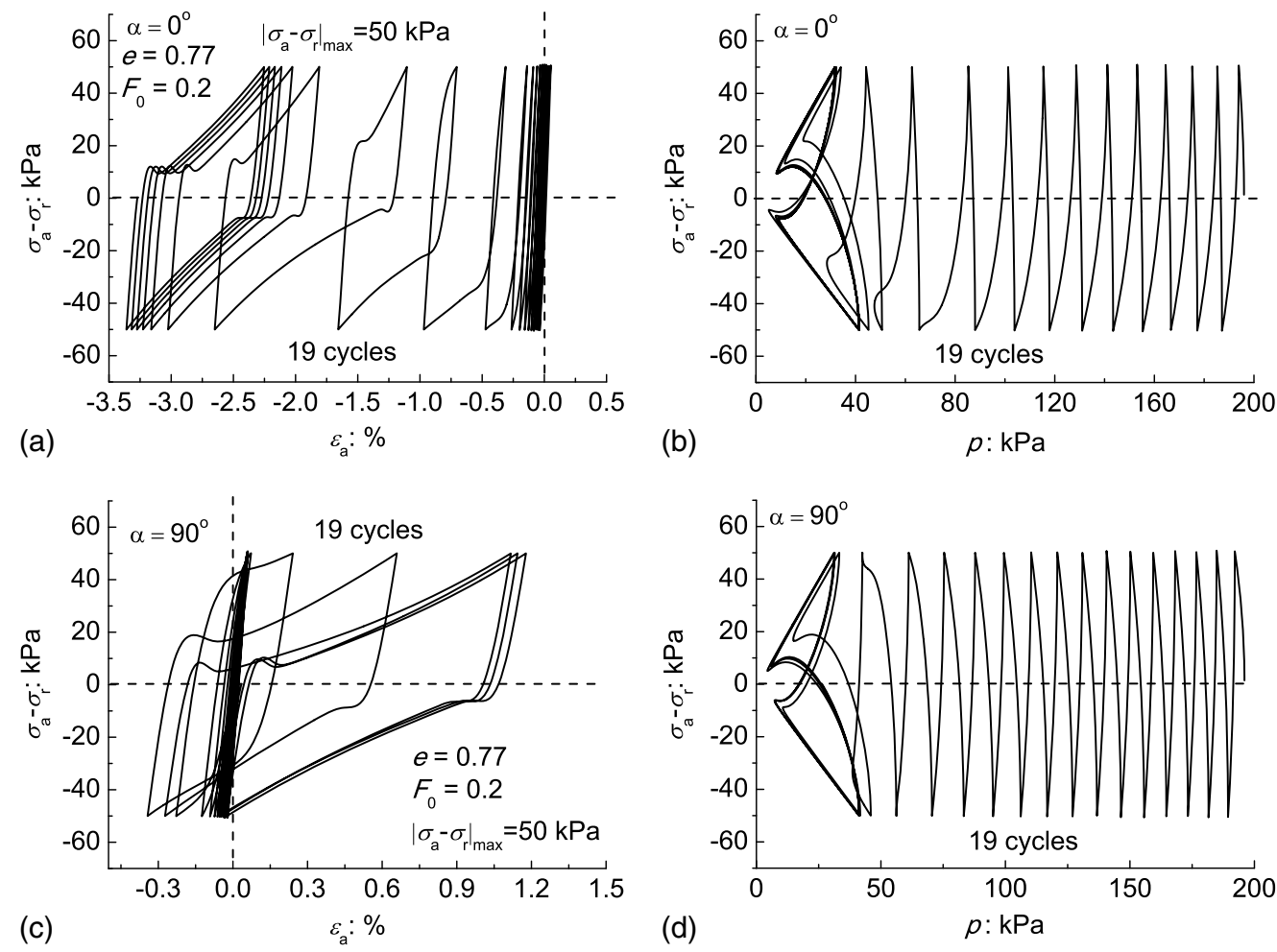

Fig. 13. Model simulations for the effect of bedding plane orientation on sand behavior in undrained cyclic triaxial test 
by depositing boiled sand in de-aired water (Ishihara et al. 1975), which is different from the preparation methods described above. Thus, $F_{0}=0.05$ is used to best fit the test results. Other parameters are the same as those shown in Table 1. The test results are obtained from Dafalias and Manzari (2004). It can be seen that the model gives good simulations for both the stress-strain relation and stress path. It should be mentioned that only $F_{0}$ is changed to simulate effect of initial fabric (i.e., different preparation methods create different initial sand fabric) on sand behavior. However, this is based on a limited number of tests. Other parameters may also need to be changed to get an accurate prediction of the effect of sample preparation on sand behavior.

Fig. 13 demonstrates the effect of bedding plane orientation on the undrained cyclic triaxial sand behavior. The initial confining pressure is $196 \mathrm{kPa}$ and $F_{0}=0.2$ for both samples. Under identical loading conditions, the effective stress path for the sample with $\alpha=0^{\circ}$ approaches the origin faster than the comparison sample with $\alpha=90^{\circ}$ [Fig. 13(b and d)]. Such sand behavior has also been observed by Miura and Toki (1984) and Oda et al. (2001) in both manmade and in situ sand samples with initially anisotropic fabric. Their studies indicate that, compared to the samples with horizontal bedding plane orientation $\left(\alpha=0^{\circ}\right)$, those with vertical bedding plane orientation $\left(\alpha=90^{\circ}\right)$ have higher cyclic undrained triaxial strength and reach initial liquefaction after more cycles. The main reason is that the samples with horizontal bedding plane orientation $\left(\alpha=0^{\circ}\right)$ show a much more contractive response in the triaxial extension side (Miura and Toki 1984; Oda et al. 2001), which is captured by our model [Fig. 13(b and d)]. In addition, the samples with $\alpha=0^{\circ}$ show faster accumulation of negative axial strain [Fig. 13(a and c)], which is also in agreement with the experimental observations (Miura and Toki 1984). Notice that cyclic mobility is observed for both samples. Note that the model gives too much preferred accumulation of negative $\varepsilon_{a}$ in Fig. 13(a) and future improvement is needed.

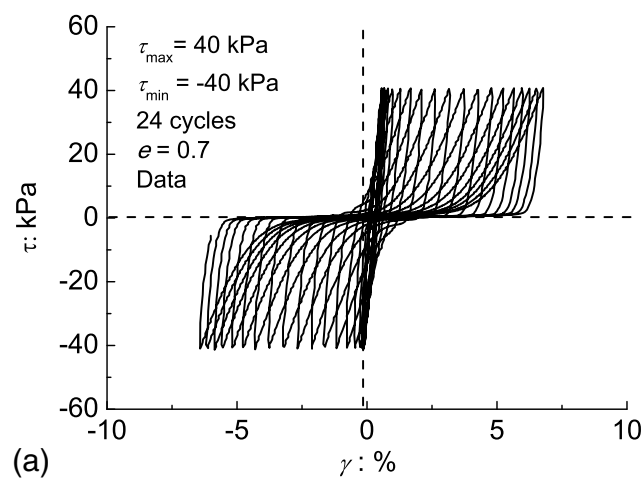

(a)

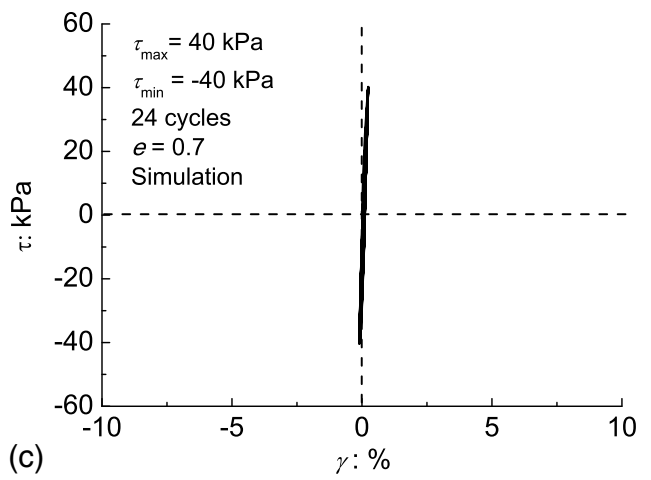

\section{Conclusion}

This paper presents a comprehensive bounding surface model to characterize the fabric effect on the behavior of sand in both monotonic and cyclic loading conditions within the framework of the anisotropic critical state theory (Li and Dafalias 2012). The model has the following key features:

1. An evolving cone-shaped bounding surface and a yield cap perpendicular to the mean stress axis are used to describe the sand behavior in constant stress ratio shear and constant mean stress compression, respectively.

2. A fabric tensor that describes the geometrical properties of void spaces of a granular assembly is employed in the model. It is assumed to evolve with both plastic shear and volumetric strains. In a shear-dominated loading, the fabric tensor will eventually become codirectional with the loading direction tensor and reach a constant magnitude of unity at the critical state. In constant stress ratio compression, the fabric tensor will finally become codirectional with the stress ratio tensor and reach a magnitude proportional to a normalized stress ratio.

3. An anisotropic variable that is defined as the joint invariant of the fabric tensor and loading direction tensor is used to model the fabric effect in the plastic hardening, plastic flow, and dilatancy of sand in constant mean stress shear, including both monotonic loading with fixed loading direction and cyclic loading.

4. The model offers a unified description to account for the effect of fabric and fabric evolution in both monotonic and cyclic loading of sand. The model predictions of sand behavior for a series of tests on Toyoura sand compare well with the test data.

While it has been shown to be able to capture the fabric effect on monotonic and cyclic sand behavior, the proposed model still contains several notable limitations which may be improved in the future:
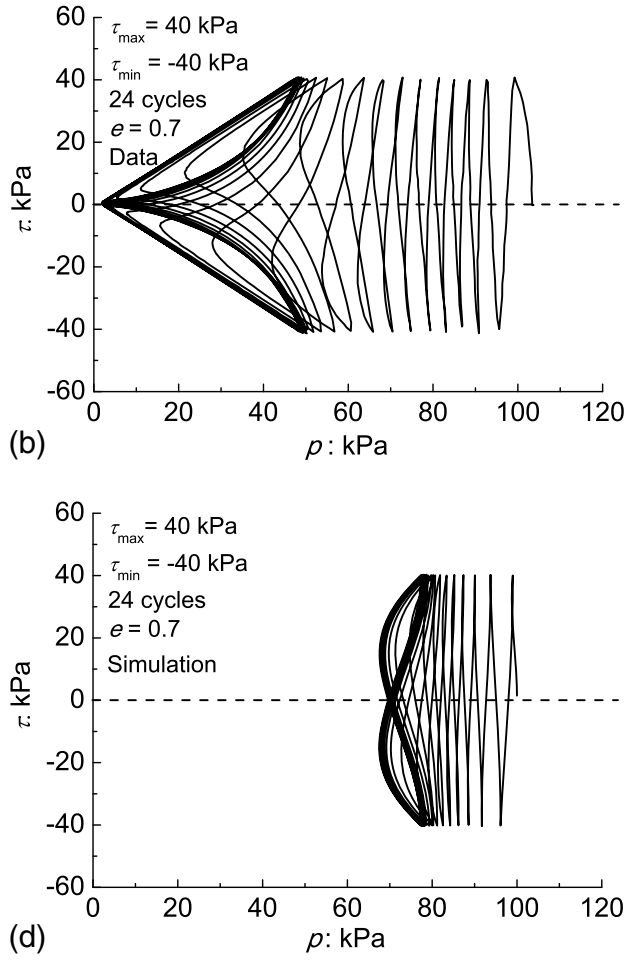

Fig. 14. Comparison between test data and model simulations for behavior of very dense Toyoura sand in undrained cyclic simple shear test 
1. The term $h_{c}$ [Eqs. (16) and (17)] is assumed to be affected by $F$ only. Such formulation is not sufficient for modeling the cyclic mobility of very dense sand. Fig. 14 shows the model simulations for the behavior of dense Toyoura sand (relative density $D_{r} \approx 80 \%, F_{0}=0.5$ ) in an undrained cyclic simple shear test (T. Kiyota, personal communication, 2013). The model gives cyclic mobility at higher mean effective stress level and lower shear strain amplitude. Indeed, past studies indicate that a plastic strain-dependent $h_{c}$ should be used to model the cyclic mobility of very dense sand (Li 2002; Ling et al. 2006; Wang and Xie 2014). For instance, in order to capture such soil response, Li (2002) and Wang and Xie (2014) assume that $h_{c}$ decreases with plastic shear strain. However, such assumption is valid for the undrained loading only, as shear modulus degradation is not observed in drained loading. A better treating technique is indeed needed to address this issue in the future.

2. The elasticity of sand is assumed isotropic in the present model. Experimental observations (e.g., Kuwano and Jardine 2002) have shown that the elastic stiffness is typically anisotropic and evolves with deformation. This can indeed be achieved by employing a fabric-dependent anisotropic elastic stiffness tensor (Cowin 1985). The evolution of anisotropic elasticity can then be naturally accounted for based on the evolution of fabric with deformation. Indeed, Lashkari (2010) showed that employment of an evolving anisotropic elastic stiffness tensor can improve the model performance in describing undrained cyclic sand response.

3. The present model cannot describe the plastic strain accumulation in sand when the stress increment is normal to the norm of the bounding surface. A third loading mechanism may be needed for modeling sand behavior under such loading conditions (Li and Dafalias 2004).

\section{Appendix I. Constitutive Equations}

The elastic relations based on Eqs. (28) and (29) can be expressed as

$$
d e_{i j}^{e}=\frac{d s_{i j}}{2 G}=\frac{p d r_{i j}+r_{i j} d p}{2 G}
$$

and

$$
d \varepsilon_{v}^{e}=\frac{d p}{K}
$$

where $d e_{i j}^{e}$ and $d \varepsilon_{v}^{e}=$ elastic deviatoric and volumetric strain increments, respectively.

Assuming that the total strain increment $d \varepsilon_{i j}=$ summation of the elastic and plastic shear strain increments $\left(d \varepsilon_{i j}=d \varepsilon_{i j}^{e}+d \varepsilon_{i j}^{p}\right)$, the following equation can be obtained based on Eqs. (5), (10) and (31)

$$
\begin{aligned}
d p & =K\left(d \varepsilon_{v}-d \varepsilon_{v}^{p}\right)=K\left(d \varepsilon_{v}-\sqrt{2 / 3} D_{1} L_{1}-\sqrt{2 / 3} D_{2} L_{2}\right) \\
& =L_{2} K_{p 2}
\end{aligned}
$$

Thus, $L_{2}$ can be expressed in terms of $L_{1}$ as below according to Eq. (32)

$$
L_{2}=\frac{K d \varepsilon_{v}-\sqrt{2 / 3} K D_{1} L_{1}}{K_{p 2}+\sqrt{2 / 3} K D_{2}}
$$

According to the additive decomposition of the total strain increment and Eqs. (3), (6), (9), (30), and (32), one has

$$
\begin{aligned}
2 G \bar{n}_{i j}\left(d e_{i j}-L_{1} \bar{m}_{i j}-L_{2} l_{i j}\right)= & K_{p 1} L_{1}+\bar{n}_{i j} r_{i j} K\left(d \varepsilon_{v}\right. \\
& \left.-\sqrt{2 / 3} D_{1} L_{1}-\sqrt{2 / 3} D_{2} L_{2}\right)
\end{aligned}
$$

The expression for $L_{1}$ can be obtained based on Eqs. (33) and (34) as below

$$
L_{1}=\frac{2 G \bar{n}_{i j}-\left(\bar{n}_{a b} r_{a b}+B K\right) \delta_{i j}}{C-\sqrt{2 / 3} B K D_{1}} d \varepsilon_{i j}=\Theta_{i j} d \varepsilon_{i j}
$$

where

$$
B=\frac{2 G \bar{n}_{i j} l_{i j}-\bar{n}_{i j} r_{i j} K \sqrt{2 / 3} D_{2}}{K_{p 2}+\sqrt{2 / 3} K D_{2}}
$$

and

$$
C=2 G \bar{n}_{i j} \bar{m}_{i j}-\sqrt{2 / 3} \bar{n}_{i j} r_{i j} K D_{1}+K_{p 1}
$$

The expression for $L_{2}$ can be obtained by substituting Eq. (35) into Eq. (33) as following

$$
L_{2}=\frac{K \delta_{i j}-\sqrt{2 / 3} K D_{1} \Theta_{i j}}{K_{p 2}+\sqrt{2 / 3} K D_{2}} d \varepsilon_{i j}=\Omega_{i j} d \varepsilon_{i j}
$$

The incremental stress-strain relation can then be written as the following based on Eqs. (6), (8), (9), (10), (33), (38), and the additive decomposition of the total strain increment

$$
\begin{aligned}
d \sigma_{i j}= & E_{i j k l} d \varepsilon_{k l}^{e}=E_{i j k l}\left(d \varepsilon_{k l}-d \varepsilon_{k l}^{p}\right) \\
= & E_{i j k l}\left[d \varepsilon_{k l}-\left(d e_{k l}^{p}+d \varepsilon_{v}^{p} \delta_{k l} / 3\right)\right] \\
= & E_{i j k l}\left[d \varepsilon_{k l}-h\left(L_{1}\right)\left(\bar{m}_{k l}+\sqrt{2 / 27} D_{1} \delta_{k l}\right) L_{1}\right. \\
& \left.-h\left(L_{2}\right)\left(l_{k l}+\sqrt{2 / 27} D_{2} \delta_{k l}\right) L_{2}\right] \\
= & {\left[E_{i j k l}-h\left(L_{1}\right) Y_{i j} \Theta_{k l}-h\left(L_{2}\right) Z_{i j} \Omega_{k l}\right] d \varepsilon_{k l}=\Lambda_{i j k l} d \varepsilon_{k l} }
\end{aligned}
$$

where $h(L)=$ Heaviside step function, with $h(L>0)=1$ and $h(L \leq 0)=0$ and

$$
E_{i j k l}=(K-2 G / 3) \delta_{i j} \delta_{k l}+G\left(\delta_{k i} \delta_{l j}+\delta_{l i} \delta_{k j}\right)
$$

$$
\begin{aligned}
& Y_{i j}=E_{i j k l}\left(\bar{m}_{k l}+\sqrt{2 / 27} D_{1} \delta_{k l}\right) \\
& Z_{i j}=E_{i j k l}\left(l_{k l}+\sqrt{2 / 27} D_{2} \delta_{k l}\right)
\end{aligned}
$$

\section{Appendix II. Evolution of the Bounding Surface}

For a nonlinear elastoplastic model, it is not necessary (or often impossible) to present an explicit expression of hardening parameter. However, an incremental of it, i.e., the evolution law, is needed to furnish a constitutive model. It is instructive to add some remarks on the bounding surface $\bar{f}_{1}$ first. In the current model, there is a preexisting $\bar{f}_{1}$ at the initial state. When the initial state is on $\bar{f}_{1}$ (e.g., a virgin loading), the initial value of $\bar{H}_{1}=$ initial $R / g(\theta)$ according to Eq. (1). If the stress state is initially inside the bounding surface, the initial $\bar{H}_{1}=$ a state variable that should be given based on the past loading history, which is essentially equal to the size of the bounding surface. Whereas the evolution of the size of $\bar{f}_{1}$ is implicitly given in the model, the derivation for the explicit 
expression of $d \bar{H}_{1}$ is provided here. The condition of consistency for $\bar{f}_{1}$ can be expressed in terms of the image stress state as below

$$
d \bar{f}_{1}=\frac{\partial \bar{f}_{1}}{\partial \bar{r}_{i j}} d \bar{r}_{i j}+\frac{\partial \bar{f}_{1}}{\partial \bar{H}_{1}} d \bar{H}_{1}=\frac{\partial \bar{f}_{1}}{\partial \bar{r}_{i j}} d \bar{r}_{i j}-\left\langle L_{1}\right\rangle r_{\bar{H}_{1}}=0
$$

where

$$
d \bar{H}_{1}=\left\langle L_{1}\right\rangle r_{\bar{H}_{1}}
$$

Eq. (43) can also be expanded as below

$$
\begin{aligned}
d \bar{f}_{1} & =\left(\frac{\partial \bar{f}_{1}}{\partial \bar{r}_{i j}}-\frac{\partial \bar{f}_{1}}{\partial \bar{r}_{m n}} \delta_{m n} \frac{\delta_{i j}}{3}\right) d \bar{r}_{i j}+\frac{\partial \bar{f}_{1}}{\partial \bar{r}_{m n}} \delta_{m n} \frac{\delta_{i j}}{3} d \bar{r}_{i j}-\left\langle L_{1}\right\rangle r_{\bar{H}_{1}} \\
& =0
\end{aligned}
$$

Since $\frac{\partial \bar{f}_{1}}{\partial \bar{r}_{m n}} \delta_{m n} \frac{\delta_{i j}}{3} d \bar{r}_{i j}=0$, Eq. (45) can be rewritten as

$$
d \bar{f}_{1}=B \bar{n}_{i j} d r_{i j}-\left\langle L_{1}\right\rangle r_{\bar{H}_{1}}=0
$$

where

$$
B=\left\|\partial \bar{f}_{1} / \partial \bar{r}_{i j}-\left(\partial \bar{f}_{1} / \partial \bar{r}_{m n}\right) \delta_{m n} \delta_{i j} / 3\right\|
$$

Combining Eqs. (3) and (46), one has

$$
r_{\bar{H}_{1}}=B \frac{\bar{K}_{p 1}}{p}
$$

\section{Acknowledgments}

The work was partly supported by Research Grants Council of Hong Kong (under RGC/GRF 623211). The authors would like to thank Dr. Takashi Kiyota of University of Tokyo, Dr. Gabriele Chiaro of University of Wollongong, and Dr. Jun Yang of Hong Kong University for sharing their cyclic test results on Toyoura sand. The constructive comments of the anonymous reviewers are also acknowledged.

\section{References}

Abelev, A. V., Gutta, S. K., Lade, P. V., and Yamamuro, J. A. (2007). "Modeling cross-anisotropy in granular materials." J. Eng. Mech., 10.1061/(ASCE)0733-9399(2007)133:8(919), 919-932.

Azami, A., Pietruszczak, S., and Guo, P. (2010). "Bearing capacity of shallow foundations in transversely isotropic granular media." Int. $J$. Numer. Anal. Methods Geomech., 34(8), 771-793.

Bauer, E., Huang, W., and Wu, W. (2004). "Investigations of shear banding in an anisotropic hypoplastic material." Int. J. Solids Struct., 41(21), 5903-5919.

Been, K., and Jefferies, M. G. (1985). "A state parameter for sands." Géotechnique, 35(2), 99-112.

Chiaro, G., Kiyota, T., De Silva, L. I. N., Sato, T., and Koseki, J. (2009) "Extremely large post-liquefaction deformations of saturated sand under cyclic torsional shear loading." 27th Int. Conf. on Soil Mechanics and Geotechnical Engineering, Alexandria, Egypt.

Cowin, S. C. (1985). "The relationship between the elasticity tensor and the fabric tensor." Mech. Mater., 4(2), 137-147.

Cui, L., and O'sullivan, C. (2006). "Exploring the macro- and micro-scale response of an idealised granular material in the direct shear apparatus." Géotechnique, 56(7), 455-468.

Dafalias, Y. F., and Manzari, M. T. (2004). "Simple plasticity sand model accounting for fabric change effects." J. Eng. Mech., 10.1061/(ASCE) 0733-9399(2004)130:6(622), 622-634.
Dafalias, Y. F., Papadimitriou, A. G., and Li, X. S. (2004). "Sand plasticity model accounting for inherent fabric anisotropy." J. Eng. Mech., 10.1061/(ASCE)0733-9399(2004)130:11(1319), 1319-1333.

De Silva, L. I. N. (2008). "Deformation characteristic of sand subjected to cyclic drained \& undrained torsional loadings and their modelling." Ph.D. thesis, Univ. of Tokyo, Japan.

E-Kan, M., and Taiebat, H. A. (2014). "On implementation of bounding surface plasticity models with no overshooting effect in solving boundary value problems." Comput. Geotech., 55(1), 103-116.

Gajo, A., and Muir Wood, D. (2001). "A new approach to anisotropic, bounding surface plasticity: General formulation and simulations of natural and reconstituted clay behavior." Int. J. Numer. Anal. Methods Geomech., 25(3), 207-241.

Gao, Z. W., and Zhao, J. D. (2013). "Strain localization and fabric evolution in sand." Int. J. Solids Struct., 50(22-23), 3634-3648.

Gao, Z. W., Zhao, J. D., and Yao, Y. P. (2010). "A generalized anisotropic failure criterion for geomaterials." Int. J. Solids Struct., 47(22-23), 3166-3185.

Gao, Z. W., Zhao, J. D., Li, X. S., and Dafalias, Y. F. (2014). "A critical state sand plasticity model accounting for fabric evolution." Int. J. Numer. Analyt. Meth. Geomech., 38(4), 370-390.

Guo, N., and Zhao, J. D. (2013). "The signature of shear-induced anisotropy in granular media." Comput. Geotech., 47(1), 1-15.

Ishihara, K., Tatsuoka, F., and Yasuda, S. (1975). "Undrained deformation and liquefaction of sand under cyclic stresses." Soils Found., 15(1), 29-44.

Kaliakin, V. N. (2003). "An assessment of the macroscopic quantification of anisotropy in cohesive soils." Proc., 1st Japan-U.S. Workshop on Testing, Modeling, and Simulation, Boston, MA, 370-393.

Kiyota, T., Sato, T., Koseki, J., and Abadimarand, M. (2008). "Behavior of liquefied sands under extremely large strain levels in cyclic torsional shear tests." Soils Found., 48(5), 727-739.

Kuwano, R., and Jardine, R. J. (2002). "On the applicability of crossanisotropic elasticity to granular materials at very small strains." Géotechnique, 52(10), 727-749.

Lashkari, A. (2010). "A SANISAND model with anisotropic elasticity." Soil Dyn. Earthquake Eng., 30(12), 1462-1477.

Li, X. S. (2002). "A sand model with state-dependent dilatancy." Géotechnique, 52(3), 173-186.

Li, X. S., and Dafalias, Y. F. (2002). "Constitutive modeling of inherently anisotropic sand behavior." J. Geotech. Geoenviron. Eng., 10.1061/ (ASCE)1090-0241(2002)128:10(868), 868-880.

Li, X. S., and Dafalias, Y. F. (2004). "A constitutive framework for anisotropic sand including non-proportional loading." Géotechnique, 54(1), $41-55$.

Li, X. S., and Dafalias, Y. F. (2012). "Anisotropic critical state theory: Role of fabric." J. Eng. Mech., 10.1061/(ASCE)EM.1943-7889.0000324, 263-275.

Li, X. S., and Li, X. (2009). "Micro-macro quantification of the internal structure of granular materials." J. Eng. Mech., 10.1061/(ASCE) 0733-9399(2009)135:7(641), 641-656.

Li, X. S., and Wang, Y. (1998). "Linear representation of steady-state line for sand." J. Geotech. Geoenviron. Eng., 10.1061/(ASCE)1090-0241 (1998)124:12(1215), 1215-1217.

Ling, H. I., and Yang, S. (2006). "Unified sand model based on the critical state and generalized plasticity." J. Eng. Mech., 10.1061/(ASCE)0733 -9399(2006)132:12(1380), 1380-1391.

McDowell, G. R., Nakata, Y., and Hyodo, M. (2002). "On the plastic hardening of sand." Géotechnique, 52(5), 349-358.

Miura, N., Murata, H., and Yasufuku, N. (1984). "Stress-strain characteristics of sand in a particle-crushing region." Soils Found., 24(1), 77-89.

Miura, S., and Toki, S. (1982). "A sample preparation method and its effect on static and cyclic deformation-strength properties of sand." Soils Found., 22(1), 61-77.

Miura, S., and Toki, S. (1984). "Anisotropy in mechanical properties and its simulation of sands sampled from natural deposits." Soils Found., 24(3), 69-84.

Nemat-Nasser, S. (2000). "A micromechanically-based constitutive model for frictional deformation of granular materials." J. Mech. Physics Solids, 48(6-7), 1541-1563. 
Northcutt, S., and Wijewickreme, D. (2013). "Effect of particle fabric on the coefficient of lateral earth pressure observed during onedimensional compression of sand." Can. Geotech. J., 50(5), 457-466.

Oda, M., and Nakayama, H. (1989). "Yield function for soil with anisotropic fabric." J. Eng. Mech., 10.1061/(ASCE)0733-9399(1989) 115:1(89), 89-104.

Oda, M., Kawamoto, K., Suzuki, K., Fujimori, H., and Sato, M. (2001). "Microstructural interpretation on reliquefaction of saturated granular soils under cyclic loading." J. Geotech. Geoenviron. Eng., 10.1061/ (ASCE)1090-0241(2001)127:5(416), 416-423.

Oda, M., Koishikawa, I., and Higuchi, T. (1978). "Experimental study of anisotropic shear strength of sand by plane strain test." Soils Found., 18(1), 25-38.

Oka, F., Yashima, A., Tateishi, A., Taguchi, Y., and Yamashita, S. (1999). "A cyclic elasto-plastic constitutive model for sand considering a plastic-strain dependence of the shear modulus." Géotechnique, 49(5), 661-680.

Pestana, J. M., and Whittle, A. J. (1995). "Compression model for cohesionless soils." Géotechnique, 45(4), 611-631.

Pietruszczak, S. (1999). "On inelastic behavior of anisotropic frictional materials." Mech. Cohes. Frict. Mater., 4(3), 281-293.

Richart, F. E., Jr., Hall, J. R., and Woods, R. D. (1970). Vibrations of soils and foundations, Prentice-Hall, Englewood Cliffs, NJ.

Sazzad, M. M., and Suzuki, K. (2010). "Micromechanical behavior of granular materials with inherent anisotropy under cyclic loading using 2D DEM." Granular Matter, 12(6), 597-605.

Sekiguchi, H., and Ohta, K. (1977). "Induced anisotropy and time dependency in clays." Proc., 9th Int. Conf. on Soil Mechanics Foundation Engineering, Special Session 9, Tokyo, 229-238.

Soroush, A., and Ferdowsi, B. (2011). "Three dimensional discrete element modeling of granular media under cyclic constant volume loading: A micromechanical perspective." Powder Technol., 212(1), 1-16.
Sze, H., and Yang, J. (2014). "Failure modes of sand in undrained cyclic loading: impact of sample preparation." J. Geotech. Geoenviron. Eng., 10.1061/(ASCE)GT.1943-5606.0000971, 152-169.

Taiebat, M., and Dafalias, Y. F. (2008). "SANISAND: Simple anisotropic sand plasticity model." Int. J. Numer. Anal. Methods Geomech., 32(8), 915-948.

Uthayakumar, M., and Vaid, Y. P. (1998). "Static liquefaction of sands under multiaxial loading." Can. Geotech J., 35(2), 273-283.

Vermeer, P. A. (1978). "A double hardening model for sand." Géotechnique, 28(4), 413-433.

Wahyudi, S., Chiaro, G., De Silva, L. I. N., and Koseki, J. (2010). "Stressdilatancy behavior of loose sand during drained cyclic torsional shear loading." 12th Int. Summer Symp., Japan Society of Civil Engineers, Tokyo, 183-186.

Wan, R. G., and Guo, P. J. (2001). "Effect of microstructure on undrained behavior of sands." Can. Geotech. J., 38(1), 16-28.

Wang, G., and Xie, Y. (2014). "Modified bounding surface hypoplasticity model for sands under cyclic loading." J. Eng. Mech., 10.1061/(ASCE) EM.1943-7889.0000654, 91-101.

Wang, Z. L, Dafalias, Y. F., and Shen, C.-K. (1990). "Bounding surface hypoplasticity model for sand." J. Eng. Mech., 10.1061/(ASCE) 0733-9399(1990)116:5(983), 983-1001.

Yin, Z. Y., Chang, C. S., and Hicher, P.-Y. (2010). "Micromechanical modeling for effect of inherent anisotropy on cyclic behavior of sand." Int. J. Solids Struct., 47(14-15), 1933-1951.

Yoshimine, M., Ishihara, K., and Vargas, W. (1998). "Effects of principal stress direction and intermediate principal stress on undrained shear behavior of sand." Soils Found., 38(3), 179-188.

Zhao, J., and Guo, N. (2013). "Unique critical state characteristics in granular media considering fabric anisotropy." Géotechnique, 63(8), 695-704. 